Rechtsmedizin 2021 · 31:263-279

https://doi.org/10.1007/s00194-021-00520-6

Angenommen: 9. Juni 2021

Online publiziert: 16 . Juli 2021

๑) Springer Medizin Verlag GmbH, ein Teil von Springer Nature 2021

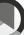

\title{
Die Jahrestagungen der Deutschen Gesellschaft für gerichtliche Medizin/ Rechtsmedizin im Spiegel der Geschichte
}

\author{
Von Meran bis München
}

\author{
K. Albrecht ${ }^{1,2} \cdot$ M. Windgassen ${ }^{3} \cdot$ S. Hartwig ${ }^{3}$ \\ 'Brandenburgisches Landesinstitut für Rechtsmedizin (BLR), Potsdam, Deutschland \\ ${ }^{2}$ Medizinische Hochschule Brandenburg (MHB), Neuruppin, Deutschland \\ ${ }^{3}$ Institut für Rechtsmedizin, Charité - Universitätsmedizin Berlin, Berlin, Deutschland
}

Zusammenfassung

In diesem Beitrag wird die chronologische Entwicklung der Jahrestagungen der Deutschen Gesellschaft für Rechtsmedizin (DGRM) von 1905 bis 2021 thematisiert. Dabei werden sowohl die medizinischen und wissenschaftlichen Aspekte als auch die spezifischen Probleme des Faches im Kontext der jeweiligen politischen und gesellschaftlichen Strukturen dargestellt und die Tagungskultur skizziert.

\section{Schlüsselwörter}

Fachtagung · Politik · Gesellschaftliche Strukturen · Internationale Kooperation · Erinnerungskultur

In der Historie der Jahrestagungen der Deutschen Gesellschaft für gerichtliche Medizin/Rechtsmedizin spiegelt sich die medizinische und wissenschaftliche Entwicklung von mehr als 100 Jahren wider, die insbesondere durch die politischen und gesellschaftlichen Strukturen des 20. Jh., von bedeutenden medizinischen Fortschritten, als auch von epochalen Katastrophen geprägt war. In der Geschichte der gerichtlichen Medizin erlebte das Fach in Bezug auf seine Eigenständigkeit und freie Entfaltung bereits vor der Gründung der Fachgesellschaft Einschränkungen, da Fächer wie die pathologische Anatomie und auch die öffentliche Gesundheitspflege ihre fachliche Stellung unterstrichen bzw. zur eigenen Selbstständigkeit drängten. Umso wichtiger war es, die Merkmale des eigenen Faches zu "konkurrierenden“ Nachbardisziplinen herauszuarbeiten und nach außen darzustellen.
Im Rahmen der Sektionssitzung "Gerichtliche Medizin" der 76. Naturforscherversammlung 1904 in Breslau, die sich bereits 1886 konstituierte, stellte Georg Puppe (1867-1925), Lehrstuhlinhaber für gerichtliche Medizin in Königsberg und Breslau, den Antrag zur Bildung einer ständigen gerichtsärztlichen Vereinigung. Gemäß dem Inhalt der Satzung der neu gegründeten Gesellschaft wurden die Tagungen zunächst weiter im Rahmen der Naturforscherversammlungen abgehalten, in den bereits in Vorjahren regelmäßig Vortragssektionen für gerichtliche Medizin integriert waren. Diese Sitzungen wurden jedoch zeitweilig mit anderen Fachgebieten wie der Hygiene und der Unfallheilkunde geteilt. In den nachfolgenden Tagungseröffnungsreden wurden die jeweiligen Entwicklungen des Faches, diezeitlich bedingten Schwerpunkte der Wissenschaft und die politischen Herausforderungen, mit- 


\section{Gerichtliche Medizin.}

\author{
10.
}

Verhandlungen der I. Tagung der Deutschen Gesellschaft für gerichtliche Medizin in Meran, 25.-28. September 1905.

Erster Sitzungstag.

Konstituierende Versammlnng der Gesellschaft für gerichtliche Medizin.

Herr Strassmann-Berlin: M. H.! Wie bekannt hat die Sektion für gerichtliche Medizin der Naturforseher-Versammlung auf der vorjährigen Tagung besehlossen, sich als deutsche Gesellschaft für gerichtliche Medizin zu konstituieren. Es ist in der betreffenden Sitzung ein vorläufiger Vorstand gewählt worden, bestehend aus den Herren Ipsen, Kratter, Lesser, Puppe, Ungar und mir. Dieser Vorstand ist zusammengetreten und hat mich zum Vorsitzenden und Herrn Professor Puppe zum Schriftü̈hrer gewählt. Infolge dessen ist mir die hohe Ehre zuteil geworden, die erste Sitzung der deutschen Gesellschaft für gerichtliche Medizin zu eröffnen.

M. H., als wir es unternommen haben, eine deutsche Gesellschaft für gerichtliche Medizin zu gründen, da haben wir nichts getan, was etwa neu und ohne Beispiel wäre. Schon seit einer Reihe von Jahren bestehen und blühen in einer Anzahl anderer Länder nationale Gesellschaften der gleichen Art.

Wenn ich absehe von der Medico-Legal Society zu New York, so hat in Europa sich zuerst die französische gerichtliche Medizin bereits vor 38 Jahren Zentrum geschaffen, die Société médecine légale de France, eine Geselloch fot von anerkanntem Ruf und Ansehen. Seit 16 Jahren wirkt in Belgien die Gesellschaft gleichen Namens in ausserordentlich verdienstvoller Weise. Vor etwa 4 Jahren ist in England eine gerichtliche medizinische Gesellschaft gegründet worden, deren erster, wertvoller und lesenswerter Verhandlungsbericht kürzlich erschienen ist. Wenn die italienisehen Kollegen, die vor 7 Jahren ebenfalls eine Gesellschaft für gerichtliche Medizin regründet haben, seit ihrer ersten dameligen Tarung niebt wieder an obe zweiten zusammengekommen sind, so liegt dies an persönlichen Rivalitäten, die sich dort unter den Vertretern 'des Faches abgespielt haben, Störungen, die unserer Gesellschaft stets fern bleiben mögen und, wie wir hoffen dürfen, stets ern bleiben werden.

Es ist also nicht etwas besonderes Kühnes, was wir unternommen haben, als wir ebenfalls eine Gesellschaft für geriehtliche Medizin gründeten. Was in Frank-

Vierteljahrsechrift t. ger. Med. u. off. San-Wesen. 3. Folge. XXXI. 2. 14

unter in persönlichen Schilderungen des jeweiligen Vorsitzenden, aufgezeigt.

Im Spätsommer des Jahres 1905 wurde die erste konstituierende Versammlung der neuen Fachgesellschaft im Rahmen der Naturforscherversammlung abgehalten. Der erste Tagungsort war unter dem Vorsitz des Berliner Gerichtsmediziners Fritz Strassmann (1858-1940) der Kurort Meran ([5]; - Abb. 1).

Meran ist die zweitgrößte Stadt Südtirols und in diesem Teil des Bundeslandes die einzige Stadt, deren Bevölkerung sich heute etwa zur Hälfte aus italienischund deutschsprachigen Einwohnern zusammensetzt. Die Verbindung beider kultureller Kreise, ihrer zum einen alpenländischen als auch mediterranen Traditionen und die gemeinsame Stadtentwicklung prägen den Lebensstil der Kurstadt von der kulinarischen Vielfalt und der Architektur bis zur Kultur. Mitte des 19. Jh. organisierte sich Meran als klimatischer Kurort, deren prominentester Gast einige Jahre später die österreichische Kaiserin
Elisabeth (,'Sissi“, 1837-1898) ist. Die Eröffnung des ersten Kurhauses erfolgte 1874; dieses wurde in den Jahren 1913 und 1914 zum heutigen Bau erweitert. Hier sollte 2021 die Festveranstaltung anlässlich der „100. Jahrestagung der Deutschen Gesellschaft für Rechtsmedizin" (DGRM) abgehalten werden [1, 3].

\section{5-1913 Weg zur Eigenständig- keit}

\section{Tagung in Meran (25.-28. September 1905)}

Unter Vorsitz des Gerichtsmediziners Fritz Strassmann, der seit 1894 den Berliner Lehrstuhl für gerichtliche Medizin innehatte, wurde die Gründungstagung mit insgesamt 26 Referaten in Meran abgehalten [5, 6]. In seiner Eröffnungsrede thematisierte der Vorsitzende zunächst die Aspekte zur Gründung der Fachgesellschaft im Vorjahr und insbesondere deren Aufgaben und Stellung im internationalen Vergleich. Da in benachbarten Ländern und den USA bereits seit Jahren eigenständige Fachgesellschaften bestanden, sei es „.... also nicht etwas besonderes Kühnes, was wir unternommen haben, als wir ebenfalls eine Gesellschaft für gerichtliche Medizin gründeten. Was in Frankreich, Belgien, England möglich gewesen ist, das, meine ich, sollte auch in den Ländern deutscher Zunge zu erreichen sein" [14].

Da nach Strassmanns Worten die gerichtliche Medizin erst in den letzten Jahren eine würdigere Stellung erringen konnte und das Fach vielfach einer Geringschätzung und Zurücksetzung unterworfen war, wies er neben der Bedeutung der Gründung einer eigenen Fachgesellschaft insbesondere auf die eigenständige Wissenschaft hin, in dem er äußerte:

Als Ziel derselben haben wir im Wesentlichen aufgestellt: Die Förderung der wissenschaftlichen Leistungen auf unserem Gebiete. Nichts aber wird mehr dazu dienen, unser Ansehen zu heben, der gerichtlichen Medizin die Achtung zu verschaffen, die ihr noch immer nicht gewährt wird, als die Steigerung unserer wissenschaftlichen Leistungen. Wir werden auf diesem Wege mehr erreichen als durch Klagen über unsere Zurücksetzung oder Anträge auf Besserung unserer Stellung, so begründet diese auch sein mögen. (Strassmann [14])

Bezüglich des Tagungsablaufes wurde es üblich, jeweils zwei Tagungsvorsitzende zu benennen, von denen der 2 . Vorsitzende im Folgejahr das Amt des 1. Vorsitzenden im Sinne eines Kongresspräsidenten bekleidete. Die Tagung selbst wurde nach einer Einführung und einer Rede vom jeweiligen Vorstand durch diesen eröffnet, und die Berichte in einem Supplementband der Vierteljahreschrift für gerichtliche Medizin - in der Regel im Folgejahr veröffentlicht. Des Weiteren wurde die neue Satzung verlesen, die im Grundmuster an die der Deutschen Gesellschaft für pathologische Anatomie und Pathologie angelehnt war und mögliche zukünftige Änderungen in Bezug zur integrierten Sitzung innerhalb der $\mathrm{Na}$ turforscherversammlung skizzierte. Julius Kratter (1848-1926) formulierte, dass für Versammlungen der Naturforscher Orte „ins Auge gefasst" werden würden, die außerhalb von Universitätsstädten lägen. 
Für die wissenschaftliche Ausrichtung wäre es für die eigene Gesellschaft jedoch zweckmäßiger, die zukünftigen Tagungen in die nächstgelegene Universitätsstadt zu legen. Bereits nach einem Jahr konnte die neu gegründete Gesellschaft - nach einem Aufruf - eine Mitgliederzahl von „einem Viertel Tausend" benennen und einen Vermögensstand von 433 Mark verzeichnen, der sich aus bereits damalig aufgerufenen Mitgliederbeiträgen und Spenden zusammensetzte [14].

Die 2. Tagung der Gesellschaft für gerichtliche Medizin wurde in Stuttgart (Vorsitz: Strassmann) und der Folgekongress in Dresden (Vorsitz: Kratter) ausgerichtet. Bis zur 9. Tagung 1913 in Wien, der letzten vor dem 1. Weltkrieg, war ein nahezu stetiger Anstieg der Fachvorträge bei einer etwa konstanten Mitgliederzahl zu verzeichnen [5]. Im Rahmen der 4. Tagung 1908 in "Cöln" erfolgte das Eingangsreferat durch Emil Ungar (1849-1934) aus Bonn. Dieser stellte die problematische Stellung des Faches im Kontext seiner Eigenständigkeit in der Vergangenheit und der Gegenwart dar und verwies gleichsam darauf, dass es auch "noch heute" deutsche Hochschulen gäbe, in denen ein Fachvertreter völlig fehle oder es an einer genügenden Vertretung der gerichtlichen Medizin mangele. Das Wissen des Fachgebietes würde beispielsweise auch durch fachfremde Kollegen, wie derer der Geburtshilfe, gelesen. Ungar berichtet, dass sein verehrter Lehrer Veit in der Einleitung zu seinen Vorlesungen über gerichtliche Medizin ausführte:

... wer besonders begabt sei (im wissenschaftlichen Sinn), widme sich der Physiologie oder einer ähnlichen Disziplin, die dii minoris gentis ergriffen mehr die klinischen Fächer, gerichtlicher Mediziner werde nur der, welcher anderswo nicht unterzukommen wisse ... Was soll denjenigen, welcher die akademische Karriere ergreifen, will auch reizen, gerichtlicher Mediziner zu werden! $\mathrm{Ihm}$ steht keine befriedigende Lehrtätigkeit in Aussicht; er kann nicht auf eine angesehene akademische Stellung rechnen, ihm winkt auch kein materieller Ersatz [...]. Ihm bietet auch nicht die Aussicht auf Ruhm, die Triebfeder für strebende Geister, einen Ersatz! Die Zahl derer, welche für Leistungen auf dem Gebiet der gerichtlichen Medizin das richtige Verständnis haben, ist eine gar zu kleine, als dass selbst bessere Arbeiten auf diesem Gebiete in weiteren Kreisen die richtige Würdigung fänden. (Gustav Veit, 1824-1903, ab 1864 Direktor der Klinik für Geburtshilfe an der Universität Bonn; las Vorlesungen in Geburtshilfe und gerichtlicher Medizin).

Gleichwohl verwies Ungar in seinem prägnanten Einführungsreferat auf die fachlichen Fortschritte der vergangenen Jahre und die Wichtigkeit der systematischen Fortbildungen. In weiteren Referaten wurde über die Vergabe von Lehraufträgen der sozialen Medizin an Gerichtsmediziner auf Grundlage der neuen Reichsversicherungsordnung diskutiert [15].

Ein Gründungsmitglied der Fachgesellschaft - Georg Puppe -, der sich ebenfalls für die Integration der sozialmedizinischen Kompetenz in das Fach gerichtliche Medizin einsetzte, lud ein Jahr nach dem Salzburger Kongress (Vorsitz: Carl Victor Ipsen, 1866-1927, Innsbruck) im Jahr 1910 zur 6. Tagung nach Königsberg i. Pr. ein und gleichsam zur Besichtigung des seit 5 Jahren bestehenden Instituts, in dem seither im Mittel 77 Obduktionen/Jahr durchgeführt wurden. Auszugsweise führte er in seinem Eingangsreferat aus, dass neben versicherungsrechtlichen Fragen im Rahmen der Sozialmedizin auch das kriminalistische Problem - die Behandlung des Trinkers - zunehmend Beachtung fände. Puppe bedankte sich in seiner Eröffnungsansprache für die "recht erfreuliche“ Zahl der Meldungen zu Vorträgen und bemerkte bezüglich der Themenauswahl, dass die einzelnen Gruppen der gerichtlichen Medizin ziemlich gleichmäßig verteilt seien.
Diesbezüglich hoffe er, dass sich die Extensität und Intensität in ähnlicher Weise entwickeln mögen wie beim Chirurgenkongress [16].

Thematisch wurde auch auf den Folgetagungen 1911 in Karlsruhe (Vorsitz: Georg Puppe) und 1912 in Münster/Westf. (Vorsitz: Ernst Gustav Ziemke, 1867-1935, Kiel) die Motorisierung Gegenstand des wissenschaftlichen Programms. Mit der zunehmenden Verbreitung motorisierter Fahrzeuge, die sich im selben Verkehrsraum wie Pferde, Fuhrwerke, Fußgänger und Radfahrer bewegten, stieg gleichsam die Unfallwahrscheinlichkeit an. Zudem führten die (relativ) hohen Geschwindigkeiten der motorbetriebenen Verkehrsmittel zur Erhöhung der Unfallwahrscheinlichkeit und der Erlangung schwerwiegender Verletzungen [16-18].

Die 9. und vorerst letzte Fachtagung vor dem 1. Weltkrieg wurde 1913 in Wien ausgerichtet. Alexander Kolisko (1857-1918) erinnerte an die 20 Jahre zuvor, ebenfalls in Wien, abgehaltene Versammlung der Naturforscher und verwies in seiner Eröffnungsrede diesbezüglich auf den Aufschwung „unserer Wissenschaft" sowie die Reichhaltigkeit und Verschiedenheit der behandelten Themen. Die Beiträge verteilten sich neben der Morphologie auch auf die Gebiete der Psychiatrie und Toxikologie sowie auf Themen angegliederter $\mathrm{Na}$ turwissenschaften. Der 1. Vorsitzende Richard Kockel (1865-1934) aus Leipzig griff die einführenden Worte Koliskos auf und bemerkte, dass es gerade für unser Fach zweckmäßig und förderlich erscheine, die Tagung der gerichtlichen Medizin, wie all
Hier steht eine Anzeige.

$$
\text { 黛 Springer }
$$




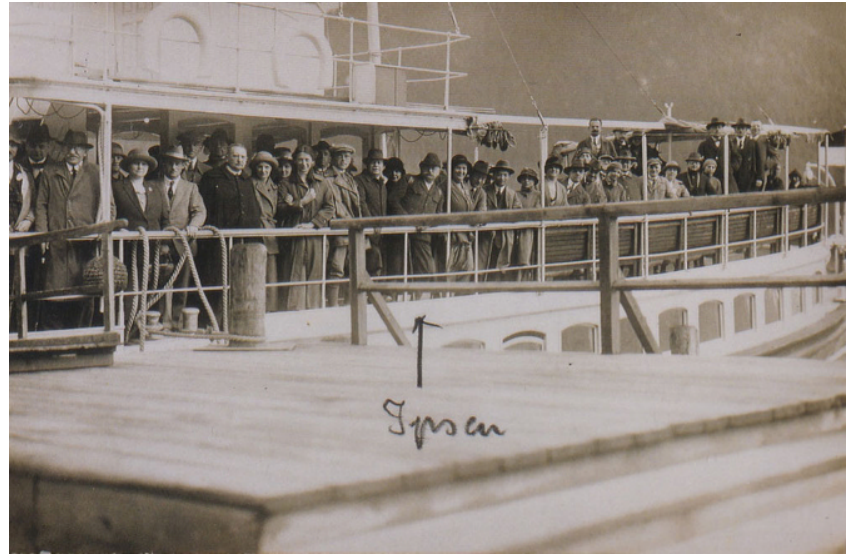

Abb. $2 \Delta$ Ausflug zum Achensee anlässlich der 13. Tagung in Innsbruck. Eine Notiz auf der Fotografie ermöglicht die Identifizierung von Carl Ipsen, der das Institut für Gerichtsmedizin in Innsbruck von 1894 bis 1927 leitete (@ Archiv DGRM, Berlin, mit freundl. Genehmigung)

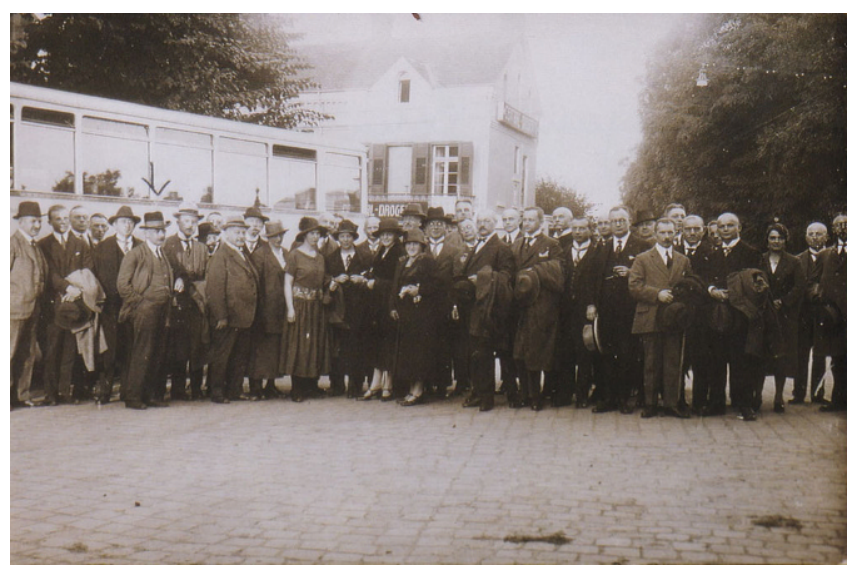

Abb. $4 \triangleleft$ Ausflug nach Neuenahr (ab 1927 Bad Neuenahr) anlässlich der 14. Tagung 1925 in Bonn. Der Pfeil deutet auf Hermann Merkel, München. (๑) Archiv DGRM, Berlin, mit freundl. Genehmigung)

die Jahre, im Rahmen der Versammlung deutscher Naturforscher und Ärzte durchzuführen und dieses auch für die Zukunft festzuhalten [19].

\section{0-1932 Depression, Hoffnung und Spezialisierungen}

Kriegsbedingt kam es auch zu Einschnitten in der medizinischen und wissenschaftlichen Landschaft. So konnte nach 1913, erst 7 Jahre später, unter dem Vorsitz von Julius Kratter im September 1920 die erste Nachkriegstagung als 10. Tagung der Deutschen Gesellschaft für gerichtliche Medizin im südhessischen Nauheim ausgerichtet werden. Der kriegsbedingt um etwa zur Hälfte gesunkenen Mitgliederzahl entsprechend weniger Vorträge als noch in Wien im Jahr 1913 gehalten. Die Diskussion um die Erweiterung des Fachgebietes wurde nach 1908 neu geführt und die Gesellschaft nach Neuantrag Puppes in „Deutsche Gesellschaft für gerichtliche und soziale Medizin" umbenannt [20].

In der mittelbaren Nachkriegszeit führte die Ausrichtung der 11. Tagung im Jahr 1921 nach Erlangen, wo der Anteil der Mitglieder und Vorträge im Gegensatz zum Vorjahr zumindest keine rückläufigen Zahlen aufwies. Da in dieser Zeit und nachfolgend die Naturforscherversammlung nur alle 2 Jahre ausgerichtet werden sollte, wurde beschlossen, die eigene Tagung im Wechsel auch mit den Tagungen der Medizinalbeamten vorzunehmen. Abweichend von den Vorjahren erfolgte die Veröffentlichung des Tagungsberichts nunmehr in Deutsche Zeitschrift für die gesamte gerichtliche Medizin, die die „Vierteljahresschrift" als Publikationsorgan ablöste. In Erlangen erfolgte unter der Einführung von Hans Molitoris (1874-1972) und unter dem Vorsitz von Theodor Lochte (1864-1953,
Göttingen) zusammen mit Vertretern der Pathologie die Diskussion um die Etablierung von Verwaltungssektionen bei unklaren Todesfällen, was jedoch aufgrund von Uneinigkeiten letztlich nicht zu einem gemeinsamen Ergebnis führte [21].

Bereits im Jahr 1922, im Rahmen der 12. Tagung in Leipzig (Einführender: Richard Kockel; 1. Vorsitzender: Fritz Reuter, 1875-1959, Graz), konnten 50 Vorträge vermeldet werden, welches bis dahin die höchste Zahl von Referaten aller bisherigen Fachveranstaltungen darstellte und die Wissenschaft in diesen schwierigen Zeiten gewissermaßen als eine Art "Hoffnungsschimmer" für das Fach fungierte [5].

Die Folgen des 1. Weltkrieges waren auch noch Jahre später, u.a. auf dem nationalen Finanzsektor spürbar, was in der Hyperinflation des Jahres 1923 gipfelte und zu einem teilweisen Zusammenbruch des deutschen Banken- und Wirtschaftssystems führte. Vermutlich aufgrund finanzieller Engpässe erfolgte in diesem Jahr keine Fachtagung, das Jahr wurde numerisch nicht angerechnet, jedoch wurden die vorgesehenen Referate publiziert und die nächste 13. Tagung 1924 in Innsbruck ausgerichtet. Im Archiv der DGRM haben sich wenige Bilder der frühen Jahre der Fachgesellschaft erhalten. Fotografien eines Ausfluges zum Achensee (• Abb. 2 und 3) bilden den Anfang.

Die Nachfolgetagungen 1925 in Bonn (Einführender: Victor Müller-Heß, 1883-1960, • Abb. 4) und 1926 in Düssel- 


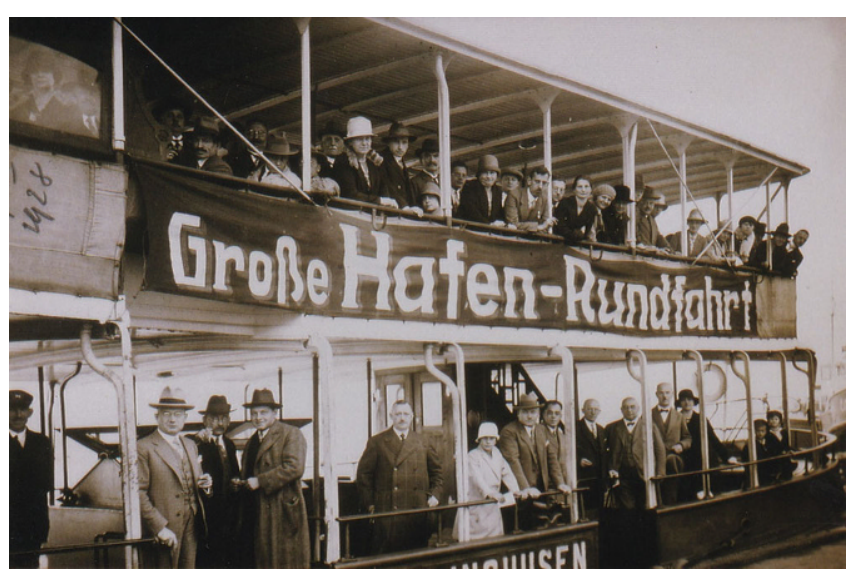

Abb. 5 ॥ "GroßeHafen-Rundfahrt" im Rahmen der 17. Tagung 1928 in Hamburg. Links unten (v. li. zwischen den Säulen): Herwart Fischer (1885-1938, Würzburg), Hermann Merkel, Walther Schwarzacher (1892-1958, Heidelberg, Wien). (@ Archiv DGRM, Berlin, mit freundl. Genehmigung)

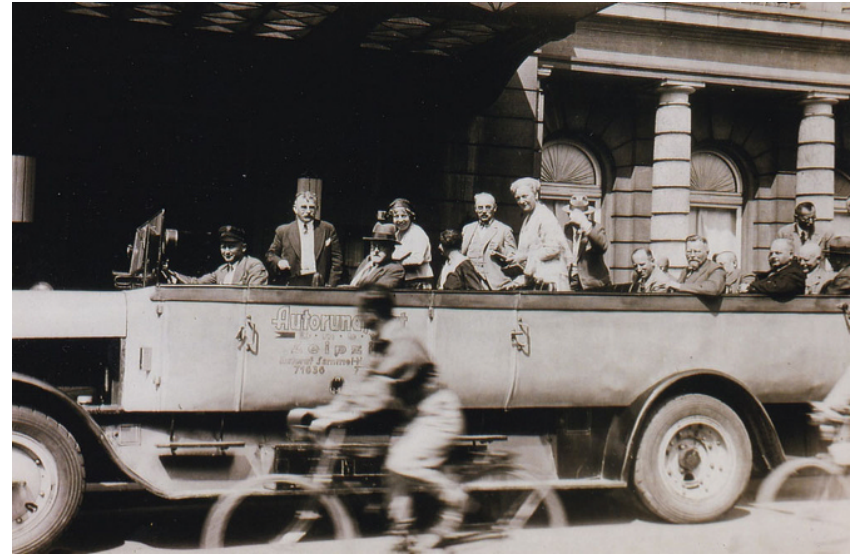

Abb. $6 \Delta$ Transfer der Tagungsteilnehmer/-innen. 20. Tagung 1931 in Leipzig (Vorsitz: Richard Kockel). Hinter dem Fahrer (v. Ii.): Hermann Merkel, Kurt Walcher (1891-1973, München, Halle-Wittenberg und Würzburg), Frau Berg, Frau Kockel, Karl Reuter (1873-1953) und Gattin. (@ Archiv DGRM, Berlin, mit freundl. Genehmigung) dorf (Einführender: Karl Berg, 1868-1936) zeigten jeweils ein facettenreiches thematisches Spektrum, wobei eine erkennbare Zahl von Vorträgen zu Schussverletzungen angemeldet wurde. Dieses war am ehesten durch Fallbeispiele von Verletzungen und Todesfällen durch die zurückgebliebenen und nicht fachgerecht benutzten Handfeuerwaffen aus dem vergangenen Krieg zu erklären. Auch Beiträge über "Selbstverstümmelungen“, um dem Dienst im Graben zu entkommen, gehörten u.a. zum kriegseigenen Spektrum der wissenschaftlichen Publikationen $[10,22$, 23].

Die 16. Tagung 1927 wurde unter Vorsitz von Victor Müller-Heß im österreichischen Graz ausgerichtet und beinhaltete einführend einen geschichtlichen und aktuellen Überblick über "alte und neue Wege in der gerichtlichen Medizin". Hermann Merkel (1873-1957) aus München referierte in seinem Eingangsvortrag über die Organisation zwischen gerichtlichem Mediziner und Gerichtschemiker in Form eines ständigen Gedankenaustausches zwischen Prosektor und Analytiker, der auch noch heute einen unverzichtbaren Bestandteil einer zielführenden forensischen Arbeit darstellt. Die verschiedenen Meinungsaspekte wurden im Anschluss an den jeweiligen Vortrag diskutiert und auch der Diskussionsteil in Form von "Wechselreden“ in den Tagungsberichten publiziert [24]. Die nachfolgenden Jahre, auch im Kontext der 17. Tagung 1928 in Hamburg (Vorsitz: Salomon Schön- berg, 1879-1958, Basel) und der Folgetagung in Heidelberg (Vorsitz: Willy Vorkastner, 1878-1931, Greifswald), standen im Zeichen neuer fachlicher Methoden und Spezialisierungen, dieses beispielsweise in Bezug auf die Serologie und Alkohologie [25]. So stellte die Thematik „Alkoholnachweis im Urin" einen Schwerpunkt der 19. Tagung im Jahr 1930 in Königsberg dar (Vorsitz: Paul Fraenckel, 1874-1941, Berlin; [26]). Selbstverständlich durfte auch ein geselliges Begleitprogramm nicht fehlen, das je nach Tagungsort in entsprechend typischer Weise ausgerichtet wurde (D Abb. 5 und 6).

\section{3-1940 Ausschaltung, Bruch und Anpassung}

Im Zuge des politischen Systemwechsels wurden neue Gesetze erlassen, die den Machtanspruch der Nationalsozialistischen Deutschen Arbeiterpartei (NSDAP) umsetzen konnten. Ein Einschnitt in die Entwicklung der Wissenschaften ergab sich u.a. durch den Erlass des Gesetzes zur Wiederherstellung des Berufsbeamtentums von 1933, das erlauben sollte, jüdische und politisch andersdenkende Beamte aus dem Dienst zu entfernen, in der Folge auch Gelehrte betraf und insofern einen Verlust geistiger Vermögenswerte bedeutete $[2,7]$. Das kurz zuvor erlassene Ermächtigungsgesetz hatte die Grundlage für diese und andere die Gesellschaft grundsätzlich verändernde Erlasse und Gesetze bereitet. In der Zeitschrift für Medizinalbeamte und Krankenhausärzte lässt Ernst Gustav Ziemke im Auftrag mitteilen, dass die anberaumte Tagung der Deutschen Gesellschaft für gerichtliche und soziale Medizin 1933 in München abgesagt wird. Vorausgegangen war, dass Martin Nippe (1883-1940) aus Königsberg auf der Vorjahrestagung in Wiesbaden 1932 als Vorsitzender, Hermann Merkel (1873-1957, München) im Hinblick auf die nachfolgende Tagung in München als Stellvertreter und Ziemke als Geschäftsführer benannt wurden. „Autoritatives Drängen“ und „örtlich gestellte Anträge" seien die Gründe für den Ausfall der 22. Tagung 1933 gewesen, wobei Merkel letztlich als Erfüllungsgehilfe den Auftrag erhalten habe, die Tagung "von sich aus" abzusagen. Nippe beschrieb zuvor, dass das ganze Schwanken daran liege, dass man von den einzelnen jetzigen maßgebenden Stellen keine ganz klaren Auffassungen extrahieren könne. Dass die nächste Tagung ganz anders gestaltet werden müsse, daran bestehe nach Nippes Urteil kein Zweifel $[2,5,9]$.

Die 23. Tagung, im Schrifttum teilweise noch als 22. Tagung aufgeführt, findet unter Einführung des Ziemke-Schülers Alex Schackwitz (1878-1952) 1934 in Hannover statt. Thematisch wurden teilweise der neuen politischen Ausrichtung angepasste Vorträge gehalten, wie beispielsweise "Die Entmannung gemeingefährlicher Gewohnheitsverbrecher" oder die "Natio- 


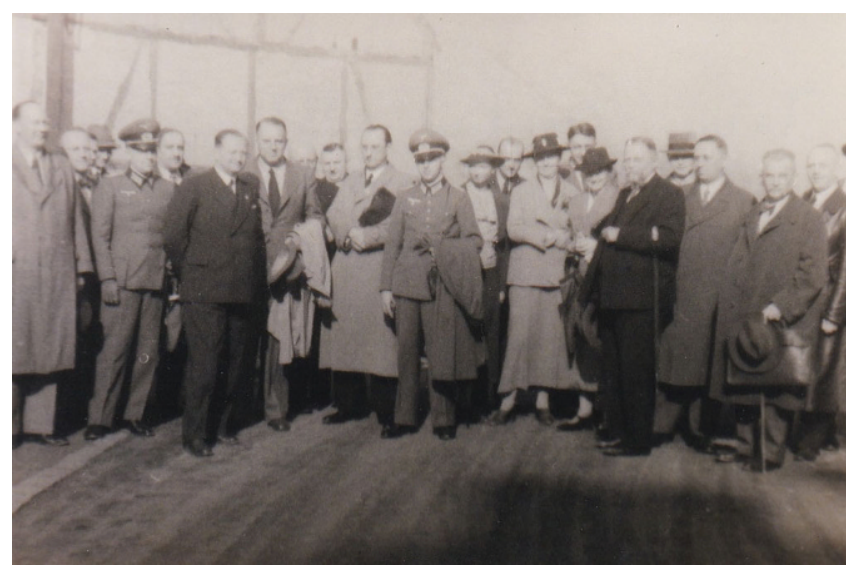

Abb. $7 \triangleleft$ Tagungsteilnehmer/-innen der 26. Tagung 1937 in Breslau. Die fortschreitende Militarisierung der Gesellschaft ist nicht zu übersehen. (@ Archiv DGRM, Berlin, mit freundl. Genehmigung)

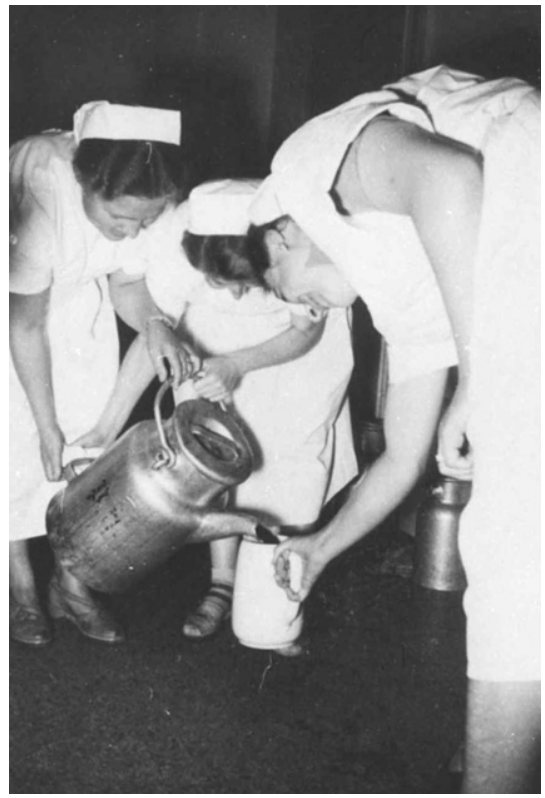

Abb. 9 \ Zünftige Pausenversorgung, 34. Tagung 1955 in Düsseldorf. (@ Archiv DGRM, Berlin, mit freundl. Genehmigung)

nalsozialistische Strafgesetzgebung". Die direkten Nachfolgetagungen zu dieser Zeit, angefangen 1935 in München bis zur 29. Tagung im Jahr 1940 in Innsbruck, die stets mindestens 30 Referate beinhalteten, standen unter erkennbar regelmäßigem Vorsitz der parteinahen Fachvertreter. Hierzu gehörten beispielsweise Friedrich Pietrusky (1893-1971, Bonn und Heidelberg) und Gerhard Buhtz (1896-1944, Breslau; D Abb. 7; [2, 27, 28])

Im Jahr 1938 wurde die 27. Tagung in Bonn unter Vorsitz von Friedrich Pietrusky international ausgerichtet, wobei der stellvertretende Kongresspräsident aus Kopenhagen anreiste.

Auf der 28. Tagung 1939 in Bad Ischl referierte Arthur Gütt (1891-1949, Arzt und
Leiter des Amts für Volksgesundheit im Reichsministerium des Inneren) über die geplante Neuordnung des gerichtsärztlichen Dienstes in Deutschland. Diese lieBe in Verbindung mit dem "Gesetz über die Vereinheitlichung des Gesundheitswesens" erkennen, dass die gerichtsärztliche Tätigkeit dem Gesundheitsamt übertragen wird: „... lässt sich von der Erkenntnis leiten, dass die gerichtsärztlichen Geschäfte zu den Aufgaben des öffentlichen Gesundheitswesens gehören" [29].

Mit der 29. „Kriegstagung" 1940 in Innsbruck endet dieser Zeitabschnitt. Noch nie, wie auf dieser letzten Tagung in der Zeit des Nationalsozialismus, sei, so Herber, die Umklammerung des Faches durch die Vollzugsorgane nazistischer Gewaltherrschaft so deutlich sichtbar geworden wie hier. Die Tagung sei diesbezüglich reich besucht gewesen. Neben den Angehörigen des Faches seien insbesondere Vertreter der Justiz und der Polizei erschienen, an deren Spitze der Staatssekretär im Reichsjustizministerium, Dr. Freisler, gewesen sei [2].

\section{1-1968 Neugründung, Trennung, Entwicklung und Aufstieg}

Mit Ende des 2. Weltkriegs im Jahr 1945 lagen viele medizinische Einrichtungen und Institute in Trümmern. Ein mühevoller Neubeginn auf vielen Ebenen war nötig; der Verlust an geistiger Kapazität war enorm. Erst 11 Jahre nach der letzten Tagung, im Jahr 1951, wurde unter dem Vorsitz von Victor Müller-Heß, erstmals in Berlin, die 30. Tagung mit der beachtlichen Zahl von 52 Vorträgen ausgerichtet. Die Eröff-

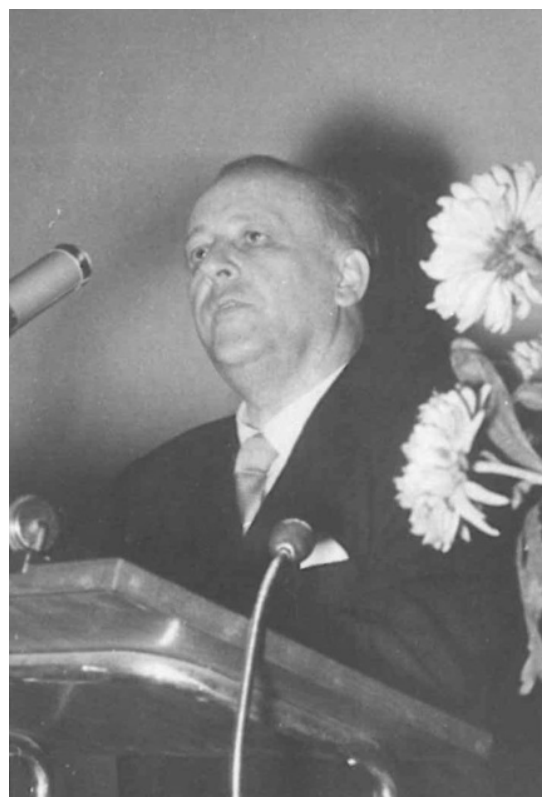

Abb. $8 \Delta$ Kurt Böhmer, Eröffnung der 34. Tagung 1955 in Düsseldorf. (@ Archiv DGRM, Berlin, mit freundl. Genehmigung)

nung des Kongresses erfolgte durch den Vorsitzenden und den Regierenden Bürgermeister von Berlin, Prof. Ernst Reuter (1889-1953). Der Tagungsbericht wird, wie in der Vorkriegszeit, jeweils im Folgejahr im Publikationsorgan Deutsche Zeitschrift für die gesamte gerichtliche Medizin veröffentlicht. In der ersten Nachkriegstagung stellten neben der forensischen Morphologie insbesondere die Thematiken der "Vaterschaftsfragen“ und auch des "Blutalkohols" inhaltliche Schwerpunkte dar. Interessanterweise wurden, auch in den Folgejahren, wenige, aber regelmäßige Beiträge zur Geschichte des Fachs präsentiert [12, 30].

Das zweite Mal nach 1935 wurde im Jahr 1952 die 31. Tagung der Fachgesellschaft, 6 Jahre nachdem die Fakultät ihren Lehrbetrieb wieder aufgenommen hatte, erneut in München unter dem Vorsitz von Wolfgang Laves (1899-1982) ausgerichtet und beinhaltete insgesamt 9 Hauptthemengebiete. Da sehr viele Beiträge eingereicht wurden, mussten die Vortragszeiten z.T. bis auf 5 Minuten gekürzt und eine "Reserveliste" erstellt werden. Die Teilnahmegebühr für die dreitägige Tagung wurde für Ordinarien, selbstständige Ärzte, Instituts- und Behördenleiter sowie Rechtsanwälte mit 20,- DM veranschlagt, Assistenten konnten für 4,- DM teilnehmen [61]. 
Die Folgetagungen der Jahre 1953-1956 in Bonn (Vorsitz: HerbertElbel, 1907-1986), Kiel (Vorsitz: Wilhelm Hallermann, 1901-1975), Düsseldorf (Vorsitz: Kurt Böhmer, 1895-1959; - Abb. 8 und 9) und Marburg (Vorsitz: Augustin Förster, 1895-1963) zeigten eine relativ konstante Zahl von Vorträgen mit jeweils über 50 Referaten in den Schwerpunkten Alkohol- und Verkehrsmedizin sowie in der Toxikologie [12, 31].

Die 36. Tagung erfolgte 1957 unter dem Vorsitz von Berthold Mueller in Heidelberg. Dieser führte in die historische Entwicklung des Instituts ein und stellte fest, dass bereits seit dem Jahr 1651 gerichtsmedizinische Gutachten von der medizinischen Fakultät erstattet worden seien. So berichtete er beispielsweise über die Frage der Ehetüchtigkeit eines Mannes, die dann bestehen würde, wenn die „Phimose" operativ beseitigt werden würde. Er führt weiter aus, dass der erste Lehrstuhl des Faches bereits um 1766 durch den Leibarzt des Kurfürsten Karl Theodor, Hubert von Harrer, besetzt wurde, nach seinem Ableben jedoch unbesetzt geblieben sei und die Lehre u.a. von Gynäkologen, Chirurgen und Internisten durchgeführt wurde, so auch vom bekannten Internisten und Gastroenterologen Adolf Kußmaul (1822-1902). Die Neugründung des Instituts sei insbesondere dem 1927 aus Graz nach Heidelberg berufenen Walther Schwarzacher (1892-1958) zu verdanken [11, 32].

Im Rahmen der Züricher Tagung im Folgejahr beschäftigten sich allein 11 Beiträge mit der Ausgestaltung und dem Aufbau des Unterrichts im Fach der gerichtlichen Medizin. So wurden neue Lehrmethoden unter Einschluss des Einsatzes von Filmen und Exkursionen vorgestellt. Auch der Umgang im Fall von sozial- und versicherungsrechtlichen Fragestellungen wurde thematisiert, wobei der Vortragende formulierte, dass er gute Erfahrungen damit gemacht habe, mit den Studenten eine Krankenkasse zu besuchen und danach mit den leitenden Persönlichkeiten der Kasse eine Art Symposium zu veranstalten [33].

Ein Jahr später wurde die 38. Jahrestagung 1959 unter dem Vorsitz von Ferdinand Wiethold (1892-1961) in Frankfurt a. $M$. ausgerichtet, der ebenfalls eine umfassende Darstellung der Entwicklung des
Frankfurter Instituts in seiner Eröffnungsrede präsentierte. Er wies darauf hin, dass Frankfurt a.M. eine höchst betriebsame und lebendige Großstadt sei, infolgedessen dem Universitätsinstitut für gerichtliche und soziale Medizin praktische Aufgaben in einem manchmal kaum noch zu bewältigenden Maße zukommen würden. Diese Fülle von wissenschaftlich anregenden und ergiebigen Beobachtungen erwünsche eine Bewältigung des Stoffes und eine forschende Auswertung. Er konstatierte: „Dass sich die meisten anderen Institute unseres Faches in ähnlichen Bedrängnissen befinden, weiß ich sehr wohl. Wenn diese Tagung dazu beträgt, einen Teil dessen, was in der Hetze des Alltags unzulänglich verarbeitet liegen bleibt, im wissenschaftlichen Gespräch und Erfahrungsaustausch zu durchdenken und in einen sinnvollen Zusammenhang zu bringen, dann wird sich das Goethewort aus „Wilhelm Meisters Lehrjahre“ bewahrheiten, welches Sie unter dem Bilde des Goethehauses auf der Umschlagseite unseres Programmheftes finden: ,Was man nicht bespricht, bedenkt man auch nicht" [34].

Die 39. und 40. Tagung wurden jeweils in Österreich, Graz 1960 (Vorsitz: Anton Werkgartner, 1890-1970) resp. Wien 1961, ausgerichtet und umfassten ein reiches Spektrum an Vorträgen über das gesamte Gebiet der gerichtlichen Medizin, wobei auf der international ausgerichteten Wiener Tagung unter Vorsitz von Leopold Breitenecker (1902-1981) insbesondere die toxikologischen und Laboratoriumsmethoden einen erkennbaren Teil des wissenschaftlichen Programms einnahmen [35, 36].

Das Leitthema „Erstickung" leitete Albert Ponsold (1900-1983) aus Münster, Westfalen, ein, der als Vorsitzender die 41. Tagung $1962 \mathrm{im}$ eigenen Institut mit einer bis dahin nie erreichten Zahl von über 80 Beiträgen eröffnete [12, 37]. Während die deutsche Medizin in den Kriegsjahren durch die weitgehende Isolierung über viele Jahre nur bedingt am wissenschaftlichen Fortschritt teilnahm, war es umso erfreulicher, dass in der Nachkriegszeit zunehmend auch wieder internationale Gäste zu den Tagungen eingeladen wurden. So konnte Ponsold Fachvertreter aus Chile, Belgien, Schweden, Italien, Ungarn, Polen, Rumänien, Bulgarien und Ju- goslawien begrüßen [37]. Die 41. Tagung ist einmal mehr Spiegel des politischen Zeitgeschehens und markiert einen Wendepunkt in der deutsch-deutschen Fachgeschichte. Mit einem Beitrag Otto Prokops (1921-2009) weist das Programm im Jahr nach dem Mauerbau im Gegensatz zu den bis dahin durchgeführten Tagungen lediglich einen Fachvertreter aus der DDR aus. Die Tagungsbeiträge aus dem Osten Deutschlands stagnieren im Gegensatz zu Beiträgen aus anderen Ostblockstaaten in den Folgejahren mit wenigen Ausnahmen bis zur politischen Wende 1989 (s. Beitrag von Preuß-Wössner et al. in diesem Heft).

In seiner Eröffnungsansprache zur 42. Tagung 1963 gab der Vorsitzende Wolfgang Laves in München einen Überblick über die Geschichte des Instituts für gerichtliche Medizin und Versicherungsmedizin der Universität München, das nunmehr seit 50 Jahren bestehe. Im wissenschaftlichen Programm wurde u.a. „Der Flugzeugunfall" aus forensischer Sicht in mehreren Vorträgen näher beleuchtet [38].

Im Folgejahr wurde die 43. Tagung in Zürich ausgerichtet. Im Rahmen der Eröffnung wurden die Sorgen der Universität zum Ausdruck gebracht - Sorgen, die für die meisten europäischen Universitäten die gleichen seien. Diese würden sich in der deutlichen Zunahme der Studenten als auch durch die "ungeheuren" Entwicklungen der Naturwissenschaften und der Medizin begründen. Inhaltlich wurde sich der traditionellen Thematik des Grenzgebietes zwischen Medizin und Recht angenommen, und Vorträge zu den „Kernfragen der ärztlichen Tätigkeit überhaupt" in das wissenschaftliche Programm aufgenommen. "Auffällig spärlich" seien indes die Vortragsanmeldungen aus dem Gebiet der Blutgruppenkunde und der forensischen Serologie. Der Eröffnungsredner führte aus, dass es diesbezüglich den Anschein mache, als ob hier eine gewisse Stagnation bzw. eine Verlagerung der Forschungstätigkeit eingetreten sei. Als Ausdruck ihrer Aktualität und Entwicklung sei die Toxikologie hingegen stark vertreten. Nachdem 6 Jahre zuvor die 37. Tagung ebenfalls schon in Zürich ausgetragen wurde, stellt der Vorsitzende Fritz Schwarz (1898-1971) abschließend fest: „Schon wieder Zürich? Ich hoffe, Sie wer- 


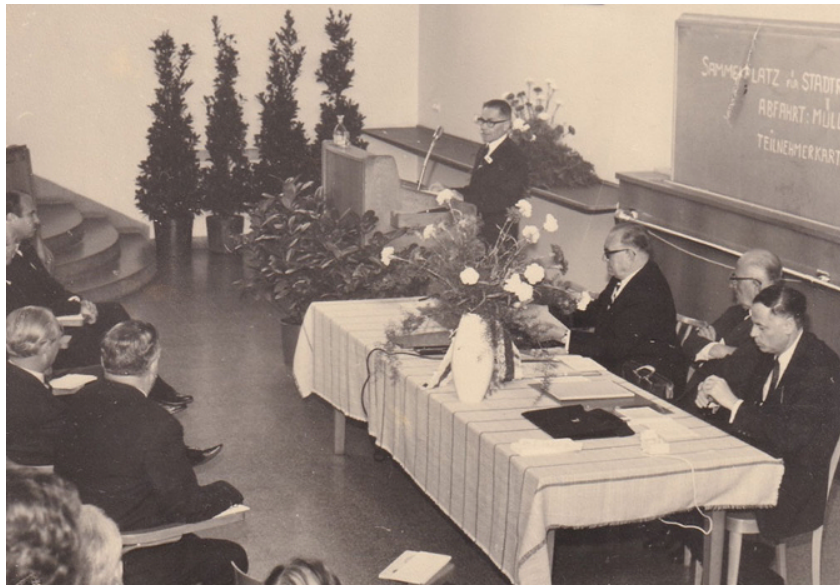

Abb. 10 ム Eröffnung der 47. Tagung 1968 in Innsbruck durch Franz Josef Holzer. (๑ Archiv DGRM, Berlin, mit freundl. Genehmigung)

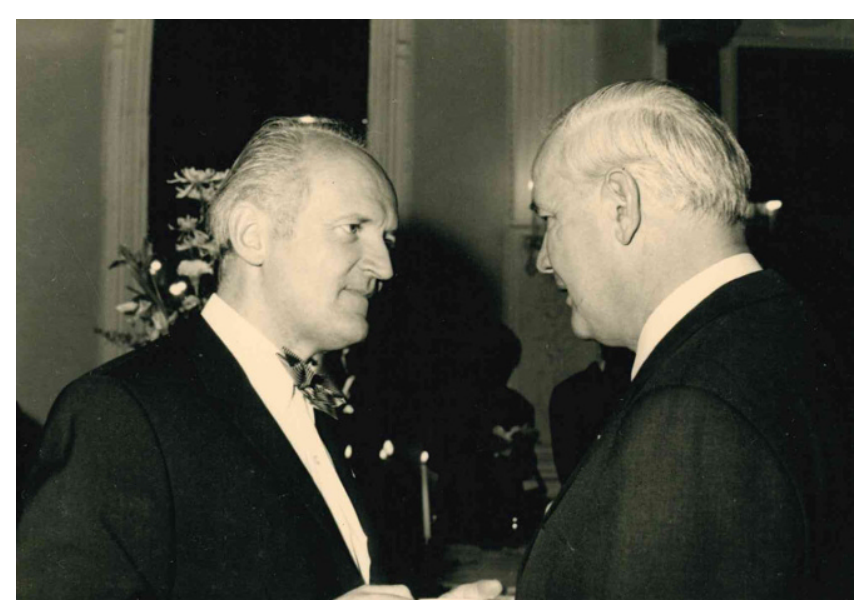

Abb. 11 ॥ "Ost trifft West" auf dem Festabend der 47. Tagung 1968 in Innsbruck. Otto Prokop (li.) und Walter Krauland (re.). (@ Archiv DGRM, Berlin, mit freundl. Genehmigung) den, wenn Sie von hier wegfahren, ausrufen: bald wieder in Zürich!“ [39].

Die 44. Tagung 1965 in Hamburg, unter dem Vorsitz von Erich Fritz (1899-1989), umfasste 90 Vorträge, wobei die Mehrzahl der Beiträge, neben toxikologischen Inhalten, mit insgesamt 20 Referaten der Thematik der "vitalen Reaktionen" zugeordnet wurde, was, vielleicht vorausschauend, den wissenschaftlichen Schwerpunkt des nachfolgenden Lehrstuhlinhabers in Hamburg andeutete $[12,40]$.

Der Sohn Fritz Strassmanns, Georg Strassmann (1890-1972), selbst Gerichtsmediziner und 1938 aufgrund seiner jüdischen Herkunft in die Vereinigten Staaten von Amerika emigriert, wurde nebst seiner Gattin auf die 45. Tagung 1966 nach Freiburg i. Br. eingeladen. Der Vorsitzende Günther Weyrich (1898-1998) referierte in seiner Eröffnungsansprache über die Geschichte der gerichtlichen Medizin in Freiburg, beginnend mit dem 18. Jh. und verwies darauf, dass es inm im Zuge des Wiederaufbaus der Universität Freiburg vergönnt gewesen sei, „das zäh und unnachgiebig verfolgte Ziel zu verwirklichen, im Jahr 1963 ein modernes Institut für gerichtliche Medizin zu errichten". Mit etwa einem Viertel der Beiträge wurde auf diesem Kongress der fachlichen Kasuistik ein großer Platz im wissenschaftlichen Programm eingeräumt [13, 41].

Kiel wurde im Folgejahr, 1967, als Ort der 46. Tagung unter Vorsitz von Wilhelm Hallermann (1901-1975) ausgewählt, da 100 Jahre zuvor, am 01.04.1867 Johan- nes Bockendahl (1826-1902, Vorgänger im Fach von Ernst Gustav Ziemke) den neu errichteten Lehrstuhl für gerichtliche Medizin in Kiel übernommen hatte. Ein Hauptthema der Tagung war die Erörterung des forensischen Beweiswertes ärztlicher Befunde und naturwissenschaftlicher Untersuchungsmethoden. Interessant erscheint die Stellungnahme Joachim Gerchows (1921-2012) aus Frankfurt/a. M., der bezüglich der Thematik "Sachverständigengutachten" formulierte, dass sich nicht jeder Fachwissenschaftler zum Gutachter eigne. Diesbezüglich sei es wünschenswert, wenn der Sachverständige beweisbare, objektivierbar nachgewiesene Sachverhalte schildern könne, jedoch ohne Verbindung mit der Rechtsfrage. Weitere Fachvorträge beschäftigten sich mit Fragen bezüglich des Zusammenhangs zwischen Schuldmaß und Alkoholgehalt des Blutes, histologischen Altersbewertungen von Blutungen, der Früherkennung eines Herzinfarktes und Möglichkeiten, die Todeszeit über fermenthistochemische Untersuchung genauer zu bestimmen. Obgleich sich die Zahl der Beiträge zum Vorjahr um etwa die Hälfte reduzierte, wurde die ganze Bandbreite der gerichtlichen Medizin und ihrer Nachbargebiete, so auch der forensischen Psychopathologie und der Versicherungsmedizin, abgebildet [42].

\section{8-1988 Umbenennung, Umbruch und Etablierung}

Die Fachgesellschaft versammelte sich zu ihrer 47. Jahrestagung 1968 in Innsbruck unter Vorsitz von Franz Josef Holzer (1903-1974, D Abb. 10 und 11).

Das Generalthema des ersten Sitzungstages beschäftigte sich mit dem Tod aus innerer Ursache, dessen Hauptreferat Walter Krauland (1912-1988) aus Berlin mit einem Vortrag über den Herztod eröffnete. Auch ausländische Teilnehmer/-innen beteiligten sich am wissenschaftlichen Programm. Ein weiterer Schwerpunkt war den Schussverletzungen gewidmet; hierzu hielt Karl Sellier (1924-1997) aus Bonn das Übersichtsreferat. Am dritten Sitzungstag eröffnete Otto Prokop ein weiteres Hauptthema, die forensische Serologie, zunächst mit einem Vortrag über das Lebensbild des Entdeckers des ABO-Systems der Blutgruppen und Nobelpreisträger, Karl Landsteiner (1868-1943). Der Tagungsvorsitzende trug im Anschluss seine persönlichen Erinnerungen an ihn vor. Ein nachfolgendes Übersichtsreferat erfolgte dann wiederum durch Prokop, der über neue Aspekte des "Immunochemical Endgrouping" und über Serumpolymorphismen berichtete. In einem vielfältigen Spektrum von insgesamt 73 wissenschaftlichen Vorträgen und ausführlichen Diskussionen erfolgte zudem auf Antrag von Wolfgang Schwerd (1924-2014) aus Würzburg am 04.10.1968 die Umbenennung der bisherigen „Deutschen Gesellschaft für gericht- 


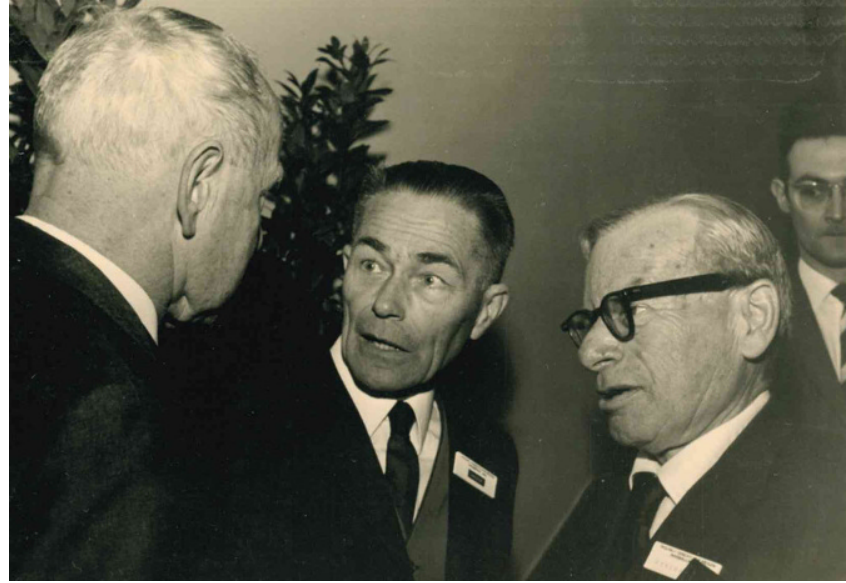

Abb. 12 ム Empfang der 48. Tagung 1969 in Berlin. Wilhelm Hallermann (li.), Franz Josef Holzer (mi.) und Georg Strassmann (re.). (๑ Archiv DGRM, Berlin, mit freundl. Genehmigung)

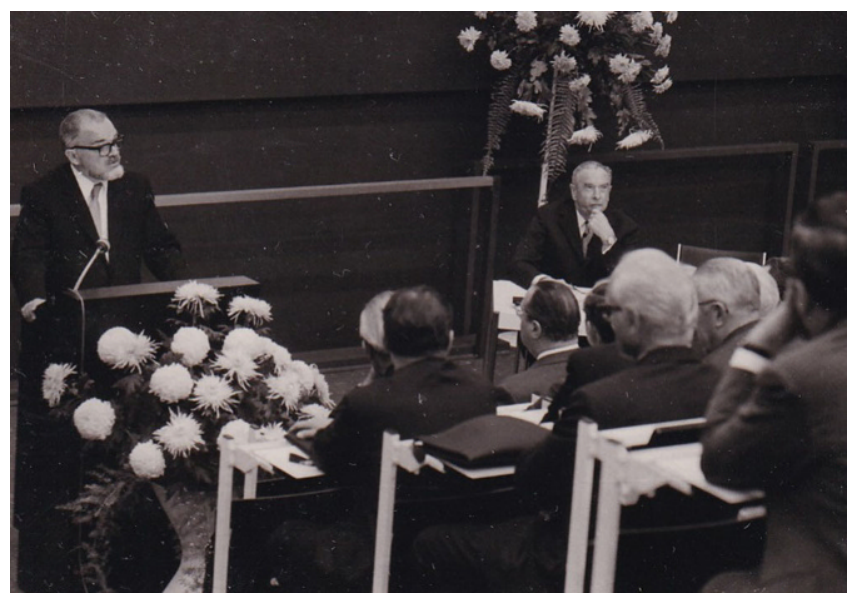

Abb. $14 \Delta$ Eröffnung der 50. Tagung 1971 in Köln durch Günter Dotzauer. (๑ Archiv DGRM, Berlin, mit freundl. Genehmigung)

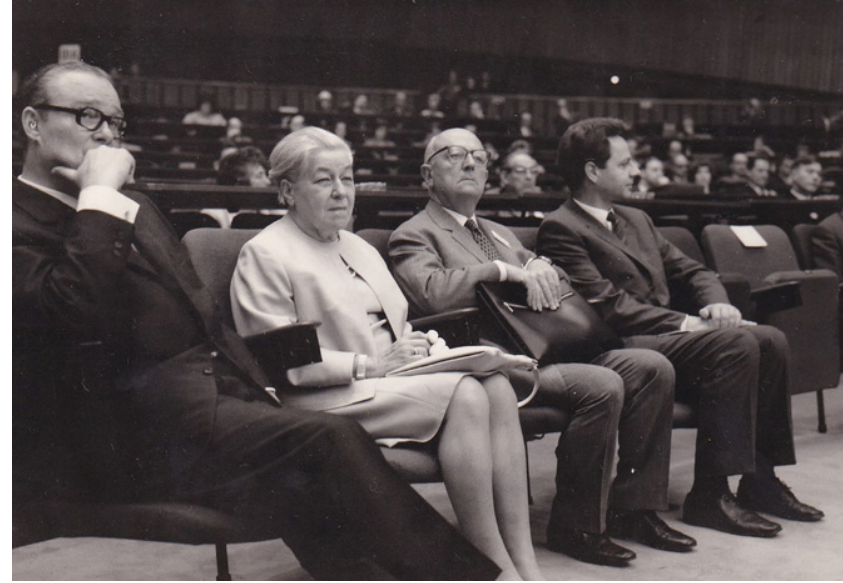

Abb. 13 A Die 48. Tagung 1969 in Berlin. Elisabeth Nau (1900-1975), erste Frau auf einem Lehrstuhl für gerichtliche und soziale Medizin (s. Beitrag von Kolbe et al. in diesem Heft). (@ Archiv DGRM, Berlin, mit freundl. Genehmigung)

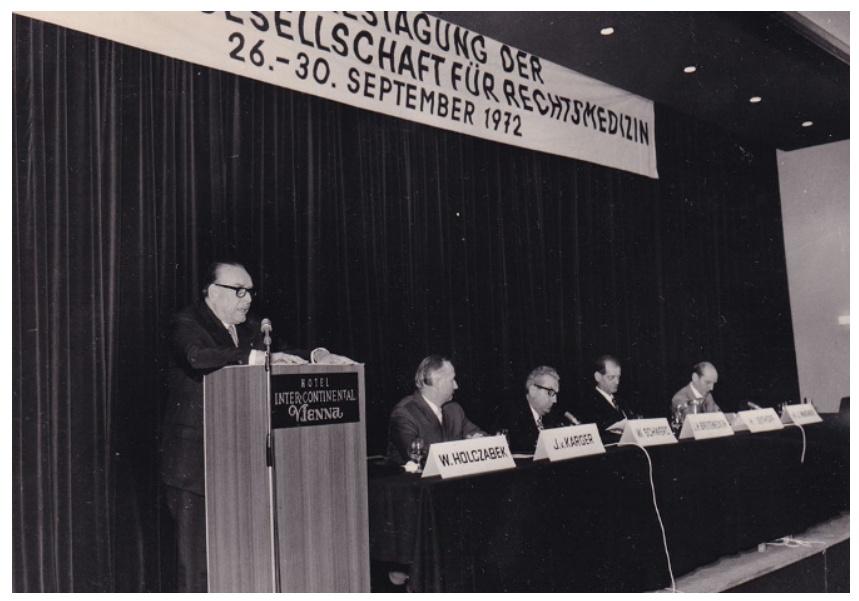

Abb. $15 \Delta$ Eröffnung der 51. Tagung 1972 in Wien, Wilhelm Holczabek (1918-2001). (@ Archiv DGRM, Berlin, mit freundl. Genehmigung) liche und soziale Medizin“ in "Deutsche Gesellschaft für Rechtsmedizin“ $[12,43]$.

$\mathrm{Zu}$ den nachfolgenden Tagungen von 1969 bis 1972 (Berlin, Vorsitz: Walter Krauland; Bern, Vorsitz: Eugen Läuppi, 1919-1999; Köln, Vorsitz: Günther Dotzauer, 1913-1990 und Wien, Vorsitz: Leopold Breitenecker, 1902-1981) wurden z.T. jeweils weit über 100 Beiträge angemeldet. Neben den klassischen Themen der Rechtsmedizin wurden auch spezielle Teilgebiete behandelt, wie beispielsweise Formen der Jugendkriminalität, radioaktive Isotopentechnik oder verkehrsmedizinische Themen wie die Unfallflucht aus verschiedenen Sichtweisen. Anzumerken ist, dass neben der Jahrestagung auch Treffen kleinerer Arbeitskreise erfolgten.
Diese Form des Wissensaustausches und der gezielten Themenbearbeitung in einem kleineren regionalen Format hat sich bis heute zu einem wertvollen Instrument weiterentwickelt ([8, 12]; • Abb. 12, 13, 14 und 15).

Spätestens seit der Zementierung der deutschen Teilung mit dem Mauerbau 1961 waren Beiträge von Kollegen aus der DDR auf den westdeutschen Tagungen aufgrund teils unüberwindbarer, politisch begründeter bürokratischer Hürden (auf Ostseite) äußerst rar. Im Jahr 1967 wurde die Gesellschaft für Gerichtliche Medizin der DDR gegründet, und 1971 die ostdeutschen Kollegen auf politischen Druck zum Austritt aus westdeutschen Fachgesellschaften - auch aus der DGRM - gedrängt (s. hierzu Beitrag von Preuß-Wössner et al. in diesem Heft). Als besondere und bemerkenswerte Geste der trotzdem gelebten deutsch-deutschen Kollegialität kann in diesem Kontext der letzte Programmpunkt der Tagung 1969 in West-Berlin aufgefasst werden: Donnerstag, den 09.10.1969, $13.00 \mathrm{Uhr}$ "Studienfahrt in das Institut für gerichtliche Medizin der Humboldt-Universität ... Abfahrt in Sonderbussen von der Kongresshalle" [62].

Wolfgang Spann (1921-2013) aus München lud im Jahr zwischen den Olympischen Spielen 1972 und der Fußballweltmeisterschaft 1974 in die bayerische Landeshauptstadt ein. In seiner Ansprache zur Eröffnung der 52. Tagung wies er auf die Notwendigkeit der Gewinnung von 


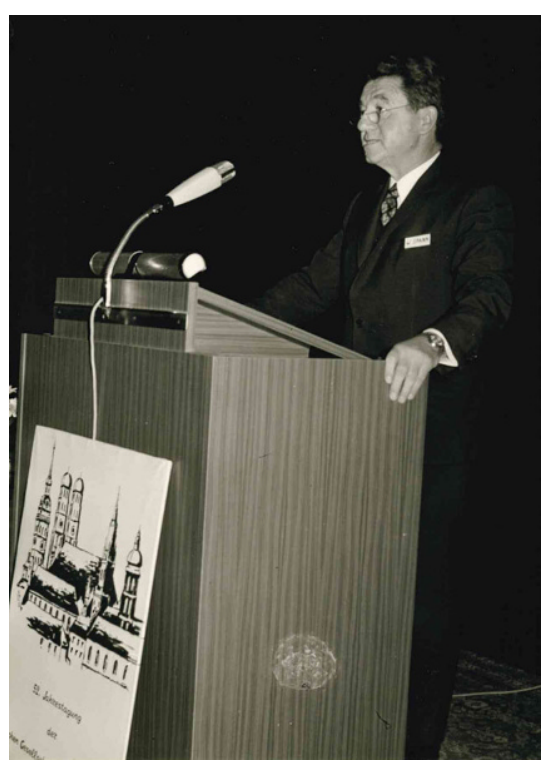

Abb. $16 \Delta$ Wolfgang Spann bei der Eröffnungsrede der 52. Tagung 1973 in München. (@ Archiv DGRM, Berlin, mit freundl. Genehmigung)

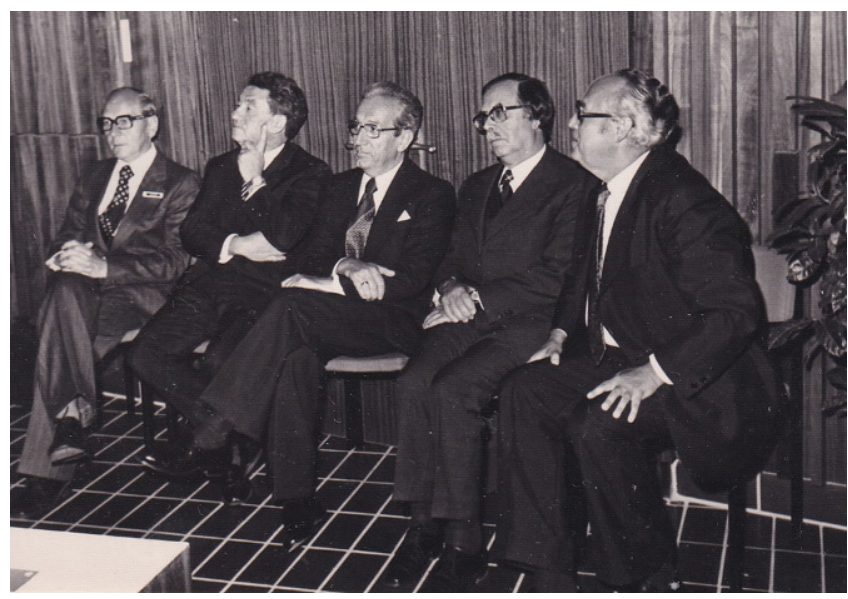

Abb. $18<$ Der

Vorstand der DGRM auf der 56. Tagung in Graz (v. li. n. re): Hans-Joachim Wagner (1924-2014, Homburg), Wolfgang Spann, Wolfgang Schwerd, Oskar Grüner (1919-2001, Gießen und Kiel), Wolfgang Maresch (৫) Archiv DGRM, Berlin, mit freundl. Genehmigung)

wissenschaftlichem und fachlichem Nachwuchs hin. Spann berichtete weiter über die Aufgaben des Münchener Instituts, wie beispielsweise über die besondere Bedeutung der praktischen Tätigkeit der körperlichen Untersuchung nach Sexualdelikten und körperlicher Misshandlung zum Zwecke der Beweismittelsicherung, da die Zahl solcher Fälle in den vergangenen Jahren deutlich angestiegen sei. Der Präsident der Fachgesellschaft, Wolfgang Schwerd, erläuterte in seiner Rede, dass sich nicht nur der Name der Gesellschaft 5 Jahre zuvor geändert habe, sondern es eine Zeit der Neuorientierung, ja des Umbruchs, gegeben habe - eine Reform. Ein Wechsel des Zeitgeistes bedinge neue Rechtsauffassungen, ständige Gesetzesänderungen würden die Rechtsmedizin, weit stärker als jedes andere medizinische Fach, beeinflussen. Schwerd wies auf die wechselvolle Geschichte des Faches hin - Blütezeiten hätten Perioden abgelöst, in denen das Fach praktisch in der Bedeutungslosigkeit versunken sei. Es sei ein großer Erfolg der vergangenen Jahre gewesen, entgegen dem Plan des Wissenschaftsrates, das Fach als Lehr- und Prüfungsfach zu erhalten ([44]; - Abb. 16).

In der Universitätsstadt Göttingen wurde im Jahr 1974 die 53. Tagung durch Steffen Berg (1921-2011) eröffnet. Hier stellte neben den klassischen Themen der Rechtsmedizin, insbesondere der Themenkomplex "Arztrecht und Ethik" einen Schwerpunkt dar - ein Gebiet, das im wissen- schaftlichen Forschungsspektrum des Vorsitzenden lag ([45]; - Abb. 17).

Die nachfolgenden Jahrestagungen fanden 1975 in Frankfurt a.M. (Vorsitz: Joachim Gerchow), 1976 in München, kombiniert mit der X. Sitzung der International Academy of Legal Medicine (IALM), unter Vorsitz von Wolfgang Spann und Erich Liebhardt (1932-1995), 1977 in Graz (• Abb. 18, Vorsitz: Wolfgang Maresch, 1918-1998), 1978 in Düsseldorf (Vorsitz: Heinz Schweitzer, 1919-1986), 1979 in Münster, Westfalen, (Vorsitz: Hans W. Sachs, 1912-2000) und als 59. Tagung 1980 in Heidelberg (Vorsitz: Georg Schmidt, 1923-2010) statt. Sie wiesen sämtlich eine hohe Zahl von Beiträgen auf, die mit 163 Referaten im Jahr 1980 einen bisherigen Höchststand zeigte [5, 12].

Krauland berichtete später, dass auf den Jahrestagungen bis zum Jahr 1980 etwa 3900 Vorträge gehalten worden seien. $\mathrm{Er}$ führte weiter aus, dass vom Beginn des Jahres 1905-1940 rund 850 Referate, zwischen 1951 und 1969 etwa 1300 Beiträge, von 1970 bis 1989 ca. 1700 Vorträge sowie zwischen 1990 und 1995 etwa 1100 wissenschaftliche Mitteilungen erschienen seien. Dieses bedeute eine Gesamtzahl von rund 5900 Beiträgen im Zeitintervall zwischen 1905 und 1995 [8]. Bis zum Jahr 1980 war ein nahezu stetiger Anstieg der Vorträge zu erkennen, woraufhin die Redezeiten auf manchen Tagungen verkürzt werden mussten. Dieses betraf auch die meist dem wissenschaftli- 
chen Programm vorangestellten Hauptreferate, welche bezüglich der Redezeit von anfangs 30-45 min auf etwa 15 min eingekürzt wurden. Schneider führt in seiner Dissertation aus, dass dies bedauerlich gewesen sei, da die Inhalte der Beiträge somit nur auszugsweise vorgestellt werden konnten und die Tagungsteilnehmer mitunter lange Zeit auf die Ergebnisse in der später veröffentlichten Originalarbeit warten mussten. Die zunehmende Zahl der angemeldeten Tagungsbeiträge führte dazu, dass zwischenzeitlich überdacht wurde, eine Aufnahmebeschränkung für Beitragsanmeldungen vorzunehmen oder zwei Tagungen im Jahr anzubieten [12].

Nachfolgende Tagungen 1981-1988

- 60. Tagung 1981 in Kiel - Vorsitz: Oskar Grüner (1919-2001),

- 61. Tagung 1982 in Würzburg - Vorsitz: Wolfgang Schwerd (1924-2014),

- 62. Tagung 1983 in Lübeck - Vorsitz: Otto Pribilla (1920-2003),

- 63. Tagung 1984 in Gießen - Vorsitz: Günter Schewe (1930-1997),

- 64. Tagung 1985 in Hamburg - Vorsitz: Werner Janssen (Jg. 1924),

- 65. Tagung 1986 in St. Gallen - Vorsitz: Hubert Patscheider (1922-2002),

- 66. Tagung 1987 in Bonn - Vorsitz: Ulrich Heifer (1930-2018),

- 67. Tagung 1988 in Erlangen - Vorsitz: Hans-Bernhard Wuermeling (1927-2019).

\section{9-1999 Wiedervereinigung, Ausbau und Festigung}

Im Jahr 1989 wurde die 68. Tagung in Salzburg unter dem Vorsitz von Gerhardt Sorgo (1935-2001) ausgerichtet, dieser folgend die 69. Jahrestagung in Köln (Vorsitz: Michael Staak, 1933-2019) und 1991 in Lausanne (Vorsitz: Hans-Rudolf Gujer, 1940-1996; [5, 8]).

Die 71. Tagung in Berlin eröffneten die Präsidenten Volkmar Schneider (Jg. 1940) und Gunther Geserick (Jg. 1938) im September 1992 - 3 Jahre nach der politischen Wende mit den einführenden Worten:

Nach 23 Jahren finden wir uns erneut in Berlin zusammen; es ist damit die dritte Jahrestagung der Deutschen Gesellschaft für Rechtsmedizin in Berlin nach dem Krieg, allerdings unter ganz neuen politischen Verhältnissen. Beide Teile Berlins wachsen vielleicht nicht ganz so rasch, wie man zunächst erhofft hat - wieder zusammen. Ganz Berlin rüstet sich darauf, Bundeshauptstadt zu werden, eine Entwicklung, die begreiflicherweise nicht ohne Schwierigkeiten abgehen kann. Wer jetzt nach Berlin kommt, wird sich der Aufbruchsstimmung nicht entziehen können. Das Leben hier ist nach dem Fall der Mauer in mancher Hinsicht sicher nicht leichter, aber um vieles interessanter geworden. In Berlin schneiden sich wieder die Ost/West-Achsen mit den Nord/SüdAchsen. Auf dem Wege nach Europa gewinnt Berlin sicher zunehmend Bedeutung. Manchen mag diese Entwicklung ängstigen, diese Angst dürfte aber unbegründet sein [63].

Konrad Händel (1909-2003, Itd. Oberstaatsanwalt, Förderer der Rechtsmedizin und Ehrenmitglied der DGRM) schrieb über die Tagung: „War die 1969er Tagung noch ein erweitertes ,Familientreffen', so fanden sich diesmal rund 600 Teilnehmer zusammen, denen ein Mammutprogramm von 265 Vorträgen geboten wurde, von den 100 nicht minder wichtigen Postern gar nicht zu reden“. Mit der Tagung wurde gleichsam ein internationales Satellitensymposium "Drogen" angeboten. Eberhard Diepgen (damaliger Regierender Bürgermeister von Berlin und Enkel des bekannten ehemaligen Medizinhistorikers Prof. Paul Diepgen an der Berliner Universität) sprach den Anwesenden ein Grußwort aus. Die Tagung selbst wurde mit einem geschichtlichen Festvortrag mit dem Titel: „Die Bedeutung der jüdischen Ärzte für die Berliner Medizin" durch Rolf Winau (1937-2006, Institut für Geschichte der Medizin der FU Berlin) sowie den Beiträgen "Blicke in die Archivbücher des gerichtsmedizinischen Institutes der Humboldt-Universität" (Geserick) und "Ein medizinhistorischer Spaziergang über die Friedhöfe von Berlin" (Schneider) feierlich eröffnet. Einen Höhepunkt des Tagungsauftaktes stellten die jeweils 15-minütigen Vorträge vergangener Lehrstuhlinhaber mit der Überschrift "Gedanken aus der Sicht eines Emeritus" dar. Vortragende: Berg (Göttingen), Gerchow (Frankfurt/a.M.), Grüner (Kiel), Holczabek (Wien), Leithoff (Mainz), Prokop (Berlin),
Schmidt (Heidelberg), Spann (München; $[46,63])$.

Die 72. Tagung 1993 in Düsseldorf war verbunden mit dem "13th Meeting of the International Association of Forensic Sciences" (IAFS) unter der Leitung von Wolfgang Bonte (1939-2000). Die gemeinsame Tagung, mit einer integrierten deutschen Sitzung, "sprengte“ manchen Rahmen, sei es durch die über 700 Vorträge in 10 Parallelsitzungen, 300 Posterpräsentationen und mehr als 1300 Teilnehmer/-innen. Während die Vorträge in Hörsälen stattfanden, mussten Plenarsitzungen und das soziale Programm in eigens dafür aufgebaute Festzelte ausweichen. Den deutschen Tagungsteilnehmern/Tagungsteilnehmerinnen wurde es ermöglicht, durch die internationale Ausrichtung des Kongresses mit Kollegen/Kolleginnen aus allen Kontinenten zusammenzukommen und sich fachlich auszutauschen. Als Überraschungseinlage des Abendprogramms erschien der USFilmstar Jack Klugman, der die Rolle des Gerichtsmediziners Dr. Quincy spielte [48]. Bezüglich des Jahres 1993 ist ergänzend zu erwähnen, dass im Vorjahr durch den Vorstand der wissenschaftlichen Fachgesellschaft eine Kommission eingerichtet wurde, die sich mit der Thematik der Gründung eines eigenen Berufsverbandes beschäftigen sollte - am 19.03.1993 fand mit 14 Mitgliedern die Gründungsversammlung des Berufsverbandes Deutscher Rechtsmediziner in Frankfurt/a.M. statt [47].

Die 73. Tagung wurde im Jahr 1994 durch das Münchner Institut unter Vorsitz von Wolfgang Eisenmenger (Jg. 1944) ausgerichtet. In einem abgedruckten Grußwort wies der Staatsminister des Innern Dr. Günther Beckstein auf die besondere Bedeutung der rechtsmedizinischen Forschung aufgrund der ansteigenden Tendenz des Drogenkonsums im Straßenverkehr hin und erwähnte die Erfolge auf dem Gebiet der forensischen Molekularbiologie vor dem Hintergrund des besorgniserregenden Kriminalitätsanstiegs und neuer Kriminalitätsformen. Den Vortragssektionen "Medizin und Recht" sowie „Public Health" am ersten Sitzungstag folgten Beitragssitzungen über klassische forensische Themen sowie Einzelsitzungen über die Verkehrsmedizin, Schuss und das "Sud- 
den Infant Death Syndrome" (SIDS). Neben dem Begrüßungsabend im Paulaner Brauhaus konnten eingeladene Gäste am Empfang der Bayrischen Staatsregierung im Kaisersaal der Residenz teilnehmen und sich am Folgetag einer Besichtigung der Königsschlösser anschließen [64].

Der im Jahr 2000 emeritierte Helmut Althoff (1935-2010), an dessen Ruhestand sich die Schließung des Instituts Aachen an der Rheinisch-Westfälischen Technischen Hochschule Aachen (RWTH) anschloss, eröffnete 1995 die 74. Tagung unter dem Motto „Von der Arztethik bis zur zweifelhaften Vaterschaft". Neben den klassischen Themen wurden, wie bereits in den Jahren zuvor erkennbar, zunehmend Referate über die Thematik SIDS präsentiert [65].

Im Jahr 1996 wurde die 75. Tagung der Fachgesellschaft in Zürich ausgerichtet. Die Eröffnung erfolgte durch Walter Bär (Jg. 1946). Trotz des "gedrängten“ wissenschaftlichen Programms sollte an der Tradition des Halbtagsausflugs festgehalten werden. Anlässlich der Jubiläumstagung erfolgten somit eine schiffsgebundene "Fortbildung" mit der MS Lindt auf dem Zürichsee und eine anschließende Festveranstaltung im Kongresshaus Zürich [66].

Unter dem Vorsitz von Annelies Klein (Jg. 1939) fand die 76. Tagung 1997 in Jena statt, wobei das wissenschaftliche Programm 130 Vorträge und 91 Posterdemonstrationen umfasste [67].

Ein Jahr später begrüßte Hans Dieter Tröger (1941-2016) als Tagungspräsident die Teilnehmer/-innen in der niedersächsischen Landeshauptstadt Hannover zur 77. Jahrestagung. Im Grußwort durfte Tröger auf 247 Beiträge, nebst geplanten 7 Workshops, wie beispielsweise zur Thematik der Immunhistochemie, der Krematoriumsleichenschau, der Begutachtung des HWS-Schleudertraumas und des Täter-Profiling verweisen. Aufgrund verschiedener Umstände musste die Tagung zeitlich gestrafft und auf den traditionellen Ausflug verzichtet werden $[49,68]$.

1999 - Frankfurt am Main. Hansjürgen Bratzke (Jg. 1946) richtete sein Grußwort an die Teilnehmenden und wies auf die Tagungsinhalte der Nahtstelle zwischen Medizin und Recht hin. Neben den allgemeinen rechtsmedizinischen Themen beinhal- tete das wissenschaftliche Programm auch Beiträge über aktuelle gesundheitspolitische Probleme. Bratzke wies zudem auf die drastischen Einschränkungen der rechtsmedizinischen Arbeitsmöglichkeiten hin, die bis zum Wegfall ganzer Institute führen würden - „Es wäre viel gewonnen, wenn der Kongress auch dazu beiträgt, dass politisch Verantwortliche die Leistungen der Rechtsmedizin auch gerade für das öffentliche Gesundheitswesen anerkennen und für solide Arbeitsbedingungen sorgen." Da die Hörsäle des Frankfurter Uniklinikums aufgrund von baulichen Maßnahmen nicht zur Verfügung standen, wurden die Tagung im Hotel Interkontinental Frankfurt und der Gesellschaftsabend im Alten Frankfurter Rathaus (Römer) ausgerichtet [69].

\section{0-2021 Digitalisierung, Zukunft und Herausforderungen}

Unter der Leitung des Tagungspräsidenten Claus Henßge (Jg. 1936) wurde die 79. Jahrestagung im Jahr 2000 in Essen eröffnet. Der Vorsitzende griff die prägnanten Worte seines Vorgängers auf und wollte mit der hiesigen Tagung gleichsam der Hoffnung Ausdruck geben, dass der Kongress nicht zu einer Abschiedsveranstaltung des eigenen Instituts und weiterer Universitätsinstitute für Rechtsmedizin des Landes Nordrhein-Westfalen werden möge. Das Präsidium hatte sich dazu entschlossen, die drei besten Posterpräsentationen jeweils mit einem Preis von 1000 DM auszuzeichnen. Die klassischen Kongressthemen wurden um die Rubrik „EDV und Bild“ ergänzt. Die Geschichte des Ruhrgebiets und der regionale Strukturwandel konnten eindrucksvoll im Rahmen der Eröffnungsveranstaltung in der ehemaligen Kompressorenhalle der 4 Jahre zuvor stillgelegten "Zeche Zollverein" nachvollzogen werden - Glück auf! [70].

Vom schweizerischen Interlaken, in dem 2001 die nachfolgende 80. Tagung (Vorsitz: Richard Dirnhofer, Jg. 1942) ausgerichtet wurde, ging es vom Kanton Bern, kaum nördlicher reichend, zur 81. Jahrestagung 2002 nach Rostock-Warnemünde. Hier begrüßte Rudolf Wegener (Jg. 1943) seine Gäste unter dem Motto: „Befund - Beweissicherung - Relevanz". Auch auf dieser Tagung wurden unterschiedliche
Workshops, wie beispielsweise zur operativen Fallanalyse (OFA) und zur Kriminologie der Gewalt angeboten. Grußworte wurden, wie bereits auf vorangegangenen Tagungen, durch den Generalbundesanwalt Kay Nehm übermittelt. Einen Höhepunkt stellten die eingeladenen Festvorträge dar. Unter anderem widmeten sich Gastredner aus Michigan und New York dem Fall O.J. Simpson resp. insbesondere dem Terroranschlag auf das World Trade Center (9/11). Bernd Brinkmann (Jg. 1939) aus Münster referierte zudem über den Fall Grams (Bad Kleinen) und Richard Dirnhofer über die Serientaten Johann „Jack" Unterwegers. Eine eigene Sitzung wurde dem Thema „Bildgebende Verfahren" gewidmet, in der dem Auditorium in 7 Vorträgen die mögliche forensische Bedeutung der schnittbildgebenden Diagnostik mithilfe von CT und MRT vorgestellt wurde. Mit dem "Konrad-Händel-Preis“, der seit 1997 von der Fachgesellschaft für besondere rechtsmedizinisch-wissenschaftliche Leistungen vergeben wird, wurden in diesem Jahr neben Gitta Mall (damals München, heute Jena) für ihre aktuelle wissenschaftliche Arbeit, auch Steffen Berg, Göttingen, Wolfgang Dürwald, Leipzig, und Otto Prokop, Berlin, für ihr wissenschaftliches Lebenswerk ausgezeichnet [50, 71].

Bernd Brinkmann lud 2003 zur 82. Tagung nach Münster ein und integrierte in sein Konzept ein englischsprachiges Fachsymposium, das „International Symposium on Forensic DNA-Technologies“. Die Möglichkeit, das Fach im globalen Kontext zu betrachten, zeigte sich in einer Zahl von nahezu 500 Teilnehmern/Teilnehmerinnen aus 30 Ländern. Der Präsident der Fachgesellschaft, Wolfgang Eisenmenger, wies in seiner Eröffnungsansprache auf die zunehmende Spezialisierung innerhalb des Faches hin, was eine Herausforderung darstelle, jedoch mit unverändertem Personalstand bewältigt werden müsse [51].

2004 -Jahr des 100-jährigen Bestehens der Deutschen Gesellschaft für Rechtsmedizin. Die Fachgesellschaft beging dieses Jubiläum mit einer separaten Festveranstaltung in Berlin. Unabhängig davon wurde die 83. Jahrestagung in Göttingen durch Klaus-Steffen Saternus (Jg. 1940) in den Räumlichkeiten der Medizinischen 
Fakultät der Georg-August-Universität abgehalten. Grund für die Auswahl des Tagungsortes war das ebenfalls 100-jährige Bestehen des Göttinger Instituts (früher: Königlich Gerichtsmedizinische Unterrichtsanstalt der Georg-August Universität zu Göttingen). Unter dem Generalthema „Rechtsmedizin und innere Sicherheit" begrüßteSaternus zahlreiche Teilnehmer/-innen aus dem In- und Ausland. Als gewisse Neuerung war erneut ein Versuch zu sehen, das wissenschaftliche Programm zeitlich zu straffen, in dem überwiegend Posterbeiträge präsentiert wurden. Der Präsident der Gesellschaft wies in seiner Eröffnungsansprache auf die schwierige Situation der rechtsmedizinischen Institute vor dem Hintergrund einer durch Sparzwänge begründeten „Profilbildung" hin. Der Festvortrag wurde von der damaligen Landesbischöfin Niedersachsens Margot Käßmann mit dem Titel "Sterben heute als Herausforderung für die Ethik" gehalten, in dem insbesondere auf die wirkungsvolle Palliativmedizin und auch auf die gebührende Beachtung von Patientenverfügungen abgestellt wurde [52].

Im Folgejahr 2005 wurde die 84. Tagung zusammen mit dem VI. International Symposium Advances in Legal Medicine (ISALM) in Hamburg ausgerichtet. An der Tagung nahmen insgesamt 550 Teilnehmer/-innen, darunter 105 japanische Kollegen/Kolleginnen, teil, die mit 370 wissenschaftlichen Beiträgen ein sehr umfangreiches Programm darboten. Der Kongress wurde vom Präsidenten Klaus Püschel (Jg. 1952) und vom Generalbundesanwalt Kay Nehm eröffnet, dem im Rahmen der Tagung die Fritz-StrassmannMedaille der DGRM zuerkannt wurde. In seiner Eröffnungsrede wies der Präsident der Fachgesellschaft Stefan Pollak (Jg. 1949) auf die schwierige Lage des Faches in Zeiten sinkender Landeszuschüsse, zunehmender Profilbildungen der Fakultäten und der Vergabe leistungsorientierter Mittel hin. Dieser Druck zwinge dazu, die Wissenschaft in den Vordergrund zu stellen, wobei ein ausgewogenes Verhältnis zur Dienstleistung geschaffen werden müsse. Nur eine forschungsstarke Rechtsmedizin im universitären Umfeld habe Zukunft. Der Wissenschaftspreis ging an Michael Thali (Jg. 1967, Bern), der für sein Forschungsgebiet der forensischen Bild- gebung (Virtopsy) ausgezeichnet wurde ein Thema, das die forensische Landschaft auch in den nächsten Jahren weiterbegleiten wird. Am 26.12.2004 hatte ein unterseeisches Erdbeben zur Entwicklung eines Tsunami an den Küsten des Indischen Ozeans geführt. Da Expertenteams aus dem deutschsprachigen Raum an den Identifizierungsmaßnahmen beteiligt waren, nahm auch dieses Thema Raum im wissenschaftlichen Programm ein [53].

Die Folgetagungen wurden als 85. Tagung 2006 in Innsbruck (Vorsitz: Richard Scheithauer, Jg. 1954) und 2007 in Mainz als 86. Tagung unter Präsidentschaft von Reinhard Urban (Jg. 1949) ausgerichtet.

Zwei Wochen, nachdem die VII. ISALMTagung in Osaka, Japan, endete, lud Jan Dreßler (Jg. 1961) 2008 nach Dresden ein. Der Präsident der Fachgesellschaft wies im Kontext des Kongressmottos „Rechtsmedizin im Wandel", nachdem er einen Überblick über die bisherigen Tagungen in Dresden gegeben hatte, auch auf die Entwicklung der Gleichberechtigung der Frauen im Fach hin, die rückblickend ein weiter Weg gewesen sei (s. hierzu den Beitrag von Kolbe et al. in diesem Heft). Die aktuelle Tagung mit etwa 200 Vorträgen und Posterbeiträgen zeuge zudem von einer großen Leistungsfähigkeit des Faches sowie dem wissenschaftlichen Engagement seiner Vertreter/-innen. Ferner wies Dreßler auf die zunehmende Diversifizierung und Spezialisierung im Fach mit der Notwendigkeit der sachgerechten Integration aller Arbeitsbereiche hin. Im Rahmen des wissenschaftlichen Programms wurden interessante Begleithemen, wie aus der Kinderradiologie und der „problemorientierten Lehre“, näher beleuchtet. Das Begleitprogramm fand seinen Höhepunkt in einem Orgelkonzert in der wiederaufgebauten Frauenkirche zu Dresden. Der Festabend wurde im Taschenbergpalais gegenüber der berühmten Semperoper und dem Zwinger ausgerichtet [54].

„Härzlig willkumme do in Basel“ - mit diesen freundlichen Worten wurden die Teilnehmer/-innen zu Beginn der 88. Tagung 2009 in Basel begrüßt. Tagungspräsident Volker Dittmann (Jg. 1951) stellte zunächst die rechtsmedizinische Struktur in der Schweiz dar und führte aus, dass es dort bislang keine Diskussionen über Institutsschließungen gegeben habe. Der
Präsident der Fachgesellschaft wies in seiner Rede darauf hin, dass Basel nicht nur die älteste Universität der Schweiz sei, sondern auch die Anfänge des Faches - der Medicina legalis - dort weit zurückreichten. Zudem stellte er die Rolle des Felix Platter im 16. und 17. Jh. dar. Die Tagung wies eine hohe Beteiligung internationaler Gäste auf. Besonders hervorzuheben war diesbezüglich auch das 20-jährige Bestehen des Osteuropavereins, das der Präsident der Fachgesellschaft zum Anlass nahm, dem Vorsitzenden Kurt Trübner (Jg. 1958, Essen) dafür zu danken. Das wissenschaftliche Programm umfasste über 200 Beiträge aus allen Bereichen der Rechtsmedizin. In der forensischen Toxikologie stellte das Thema "Spice" einen Schwerpunkt dar. Auch der klinischen Rechtsmedizin wurde im Zuge der immer häufiger vorkommenden Aufträge ein breites Podium geboten. Das Rahmenprogramm fand im Namen der Kunst statt, wobei insbesondere die Führung durch die Van-Gogh-Ausstellung im Basler Kunstmuseum Erwähnung finden muss [55].

Von Basel nach Berlin - im neu begonnenen Jahrzehnt richtete Michael Tsokos (Jg. 1967) die 89. Jahrestagung 2010 unter dem Motto "Tradition verpflichtet" aus. Auch der Präsident der Fachgesellschaft wies auf die langjährige fachliche Tradition Berlins hin, die das Identitätsempfinden der Rechtsmediziner/-innen stärken und dieses zu einer neuen Aufbruchsstimmung führen solle: „Die Besinnung auf die Wurzeln kann helfen, selbstbewusst die Kräfte zu bündeln und die Herausforderungen der Zukunft anzunehmen". Auch auf weitere Jubiläen wurde hingewiesen, wie den 100. Geburtstag des gebürtigen Berliners Konrad Händel und das 20-jährige Bestehen des Publikationsorgans Rechtsmedizin. Der Präsident stellte ferner fest: „Tradition verpflichtet - allerdings nicht zum Bewahren der Asche, sondern zum Schüren der Glut - nämlich der Glut wissenschaftlicher Weiterentwicklungen." Tsokos begrüßte die Teilnehmer/-innen 20 Jahre nach der deutschen Wiedervereinigung und zum 300-jährigen Bestehen der Charité. Die Begrüßungsveranstaltung wurde in der altehrwürdigen Kulisse der Ruine des ehemaligen Rudolf-Virchow-Hörsaals ausgerichtet. Das wissenschaftliche Programm zeigte wiederum einen internatio- 


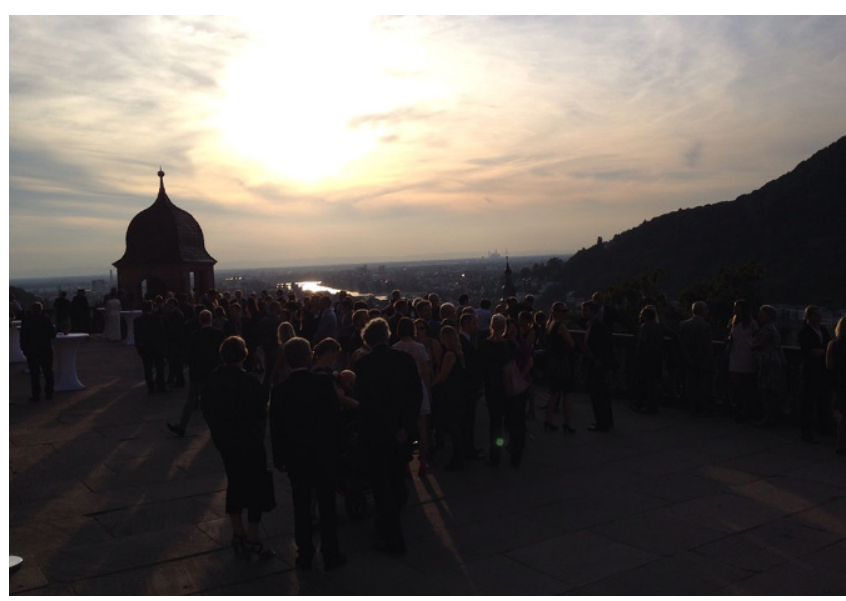

Abb. $19<$ Impressionen vom Festabend 2016. Tagungsteilnehmer/ -innen auf der Terrasse des Heidelberger Schlosses (eigene Aufnahme, Albrecht)

nalen Charakter und präsentierte etwa 300 Beiträge [56, 72].

Zwölf Jahre nach Ausrichtung des letzten Kongresses wurde die 90. Tagung der DGRM in Verbindung mit dem VIII. ISALM erneut unter dem Vorsitz von Hansjürgen Bratzke in Frankfurt/a. M. ausgerichtet. Im Rahmen der Eröffnungsveranstaltung wurde wiederholt das Thema um die stetigen Diskussionen der finanziellen Einschränkungen und den dadurch erzeugten Druck durch die ministeriellen Einsparmaßnahmen beleuchtet. Auch der bald frei werdende Lehrstuhl sei diesbezüglich als künftiges "Opfer" identifiziert worden - das Fach Rechtsmedizin müsse jedoch integraler Bestandteil der Fakultät bleiben. Eingebettet in das ISALM wurden in Gänze über 300 Beiträge, überwiegend in englischer Sprache, präsentiert. In Erinnerung geblieben ist sicher auch der Vortrag von Klaus Steffen Saternus (Göttingen), der an seinen Anfang des Jahres 2011 verstorbenen Vorgänger Steffen Berg erinnerte [57].

Ein Jahr später wurde der 91. Jahreskongress in Freiburg i. Br. in Verbindung mit dem " $1{ }^{\text {st }}$ Symposium on Interpersonal Violence in Social Proximity" durch den langjährigen Präsidenten der Fachgesellschaft Stefan Pollak ausgerichtet. Die Tagung verband zudem 2 Jubiläen - 555 Jahre Alma Mater Friburgensis und 50 Jahre Institutsgebäude der Freiburger Rechtsmedizin. Der Festabend wurde an der deutschfranzösischen Grenze in Breisach am Rhein abgehalten, nachdem zuvor eine traditionelle Sektkellerei besichtigt wurde [73].

Im Folgejahr, 2013, lud Peter Schmidt (Jg. 1958) zur 92. Tagung, ebenfalls in die Nähe der deutsch-französischen Grenze, nach Saarbrücken ein. Das abgedruckte Grußwort zur Tagung erfolgte durch die damalige Ministerpräsidentin des Saarlandes, Frau Annegret Kramp-Karrenbauer. Im wissenschaftlichen Programm, das über 260 Beiträge beinhaltete, stellte insbesondere der Themenkomplex „Klinische Rechtsmedizin“ einen erkennbaren Schwerpunkt dar [74].

Dieses Mal an der Ostseeküste fand 2014, unter Vorsitz von Britta Bockholdt (Jg. 1965) und erstmalig durch das Institut für Rechtsmedizin der Universitätsmedizin Greifswald ausgerichtet, die 93. Tagung am Veranstaltungsort Heringsdorf auf Usedom statt. Neben über 200 wissenschaftlichen Beiträgen wurden verschiedene Workshops ausgerichtet, die u.a. die aktuellen Aspekte des im Vorjahr in Kraft getretenen Patientenrechtegesetzes und die Rolle des Sachverständigen näher beleuchteten [75].

Zur 94. Jahrestagung der DGRM begrüßte Jan Dreßler 2015 etwa 400 Tagungsteilnehmer/-innen, unter ihnen Gäste aus 13 europäischen Ländern sowie aus Japan und Australien in Leipzig. Einleitende Höhepunkte waren ein Festvortrag über „600 Jahre Medizinische Fakultät der Universität Leipzig“ sowie eine Darstellung der langjährigen Institutsgeschichte. Unter dem Leitthema „Rechtsmedizin im Spannungsfeld zwischen Versorgung und Wissenschaft" wurde auf die latente Problematik, die sich aus Geld- und Personalmangel einerseits und den wachsenden Versorgungsanforderungen andererseits ergibt, ohne dabei auch dem wissenschaftlichen Aspekt Rechnung zu tragen, hingewiesen. In einer speziellen Sitzung zur Katastro- phenmedizin wurde die Arbeit des im Vorjahr verstorbenen Leipziger Lehrstuhlinhabers Wolfgang Dürwald (1924-2014) gewürdigt und in die Thematik durch Rüdiger Lessig (Jg. 1958) aus Halle eingeführt. Einen eindrucksvollen Beitrag zum Thema präsentierte der französische Gastredner Charles Agostini, der über die Identifizierung der Opfer des Germanwings-AirbusAbsturzes (Flug 9525) im März 2015 berichtete $[58,76]$.

"Rechtsmedizin 2030" - das Motto der 95. Jahrestagung 2016 in Heidelberg unter Vorsitz von Kathrin Yen (Jg. 1968), die mögliche Aspekte der zukünftigen Entwicklungen des Faches präsentierte. In einer Podiumssitzung mit Vertretern/ Vertreterinnen des Faches und der Politik wurden Fragen zur Bedeutung des Faches, zu möglichen Finanzstrategien der Versorgung und zur wissenschaftlichen Schwerpunktausrichtung diskutiert. In das wissenschaftliche Programm wurden verschiedene Workshops eingebettet, zudem Plenarsitzungen mit anderen Wissenschaftszweigen und medizinischen Fächern zur Betrachtung der Frage möglicher neuer Arbeits- und Forschungsfelder angeboten. Bezüglich der üblichen Posterpräsentationen wurde aus baulichen Gründen ein anderes Format gewählt, das eine nachträgliche Betrachtung an elektronischen Terminals ermöglichte. Der Festabend fand bei herrlichem Sonnenschein zunächst auf der Terrasse, alsdann in den Räumlichkeiten des Heidelberger Schlosses statt (- Abb. 19). Die Heidelberger Tagung ging abschnittsweise neue Wege, die mögliche zukünftige Tagungselemente beleuchtete (Format, Themen, Schnittstellen, [59]).

Eingebunden in die X. ISALM-Tagung wurde 2017 unter doppeltem Vorsitz von Stefanie Ritz-Timme (Jg. 1962, Düsseldorf) und Markus Rothschild (Jg. 1962, Köln) die 96. Jahrestagung in Düsseldorf und Köln ausgerichtet. Die Beiträge wurden bis auf wenige Ausnahmen in englischer Sprache geführt. Den Auftakt machte N. Ikeda aus Japan, der die Geschichte der "Japanese Society of Legal Medicine and Forensic Medicine" skizzierte. Neben den Workshops über Genetik, forensische Alkohologie und Dokumentationsmethoden wurden in einem Symposium die rechtlichen Probleme an der Schnittstelle zwischen 


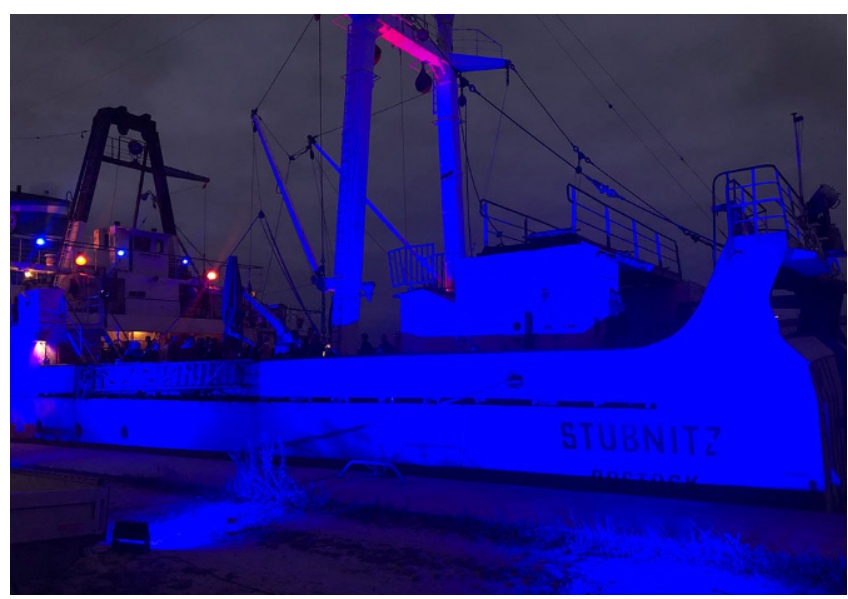

Abb. $20 \triangleleft$ Abendliche Illumination 98. Tagung 2019 in Hamburg. Festveranstaltung auf der MS Stubnitz. (Eigene Aufnahme, Albrecht)

Medizin und Recht im Vergleich zwischen Deutschland und Japan dargestellt. Eine interessante „special lecture“ wurde vom Journalisten von Lonski präsentiert, der die Rechtsmedizin aus Sicht eines Journalisten beleuchtete. Um eine gerechte Verteilung der Tagungsorte zu gewährleisten, durfte selbstverständlich ein Tagesausflug zur Domstadt Köln im Rahmen des Kultur- und Sozialprogramms nicht fehlen [77].

„Vom Universalgelehrten Alberti zum forensischen Spezialisten " - unter diesem Motto, das die enge Beziehung zwischen der Juristischen Fakultät und der Rechtsmedizin in Halle, aber auch zur Geschichte des Faches widerspiegeln sollte, lud Rüdiger Lessig 2018 zur 97. Jahrestagung nach Halle an der Saale ein. Neben dem Programm von insgesamt 88 Vorträgen und 81 Posterbeiträgen wurden begleitend Workshops, u.a. zur rechtsmedizinischen Lehre und zur Entomologie, angeboten; zudem konnte das Anatomische Institut der Universität (Meckel-Sammlung) besichtigt werden. Das Festprogramm führte die Gesellschaft zunächst nach Naumburg an der Saale mit Besichtigung des Doms und anschließend in die nahegelegene Wein- und Sektstadt Freyburg, in der im Lichthof der traditionsreichen Rotkäppchen-Sektkellerei der Festabend eingeläutet wurde [78].

Im Folgejahr, 2019, begrüßte Klaus Püschel (Jg. 1952) die Gesellschaft zur 98. Jahrestagung im historischen Hauptgebäude der Universität Hamburg - gleichsam zur Feier des 70-jährigen universitären Bestehens des Instituts für Rechtsmedizin in Hamburg. Bereits die ersten Vorträge zeigten inhaltliches Gewicht. So berich- teten die Arbeitsgruppe aus der Charité um Michael Tsokos über die rechtsmedizinische Aufarbeitung des Terroranschlags vom Breitscheidplatz, Berlin, im Dezember 2016 sowie - aus aktueller Sicht in erschreckender Weise vorausahnend eine Infektiologin aus dem Universitätsklinikum Hamburg-Eppendorf (UKE) über „Emerging Infections und andere relevante Infektionserkrankungen" und die Frage „Was kommt auf uns zu?" Die Antwort darauf wird die Welt wenige Monate später in eindrucksvoller Weise bekommen. Im Rahmen des wissenschaftlichen Programms wurden zudem verschiedene Fortbildungsveranstaltungen angeboten, wie beispielsweise zur forensischen Entomologie, zu alkoholischen Begleitstoffen, zur molekularen Autopsie und zur Molekularbiologie. Der Festabend - der Hansestadt entsprechend - erfolgte auf einem (vertäuten) Schiff. Das ehemalige Kühltransportschiff der DDR-Fischfangflotte, die MS Stubnitz, brachte das typische Flair, die Veranstaltung zünftig mit Musikund Möwenbegleitung auszurichten ([79]; - Abb. 20).

2019/2020 - „Coronavirus-disease2019“-Pandemie. Ein im Dezember 2019 erstmals bestätigter Ausbruch einer neuen Lungenerkrankung im chinesischen Wuhan. Im März 2020 erklärte die WHO die Epidemie zu einer weltweiten Pandemie, ausgelöst durch das "Severe Acute Respiratory Syndrome Coronavirus type 2" (SARS-CoV-2), mit der Folge einer globalen gesundheitlichen Notlage sowie wirtschaftlichen und sozialen Krise [4].
2020 - praktisch alle bisherig geplanten Fachveranstaltungen wurden in diesem Jahr ersatzlos abgesagt. Michael Thali aus Zürich und sein Team wagten bei sinkenden Coronainzidenzzahlen den vorsichtigen Vorstoß in der zweiten Jahreshälfte und luden zur 99. Tagung der DGRM in das schweizerische Luzern ein. Die erste Neuerung ergab sich bereits bei der Anmeldung zum Kongress - nur zuvor online angemeldete Teilnehmer/-innen waren als Kongressgäste zugelassen; Tagungsanmeldungen vor Ort waren nicht möglich. Vor der Tagung wurde ein COVID19-Schutzkonzept vorgelegt, um die potenzielle Verbreitung des Coronavirus zu verhindern und Übertragungsketten zu unterbrechen. So erfolgten Treffen der Tagungsteilnehmer/-innen beispielsweise in getrennten Gruppen, damit die Abstände zwischen den Personen gewahrt werden konnten. Die Einhaltung der Abstandsund Hygieneregeln und die Maskenpflicht (Mund-Nasen-Schutz) waren die vorrangigen Tagungsbegleiter - ein ungewöhnliches, aber notwendiges Novum. Unter dem Motto " $\mathrm{F}^{4}-$ Forensic Fit For Future" wurden trotz der eingeschränkten Lage immerhin 91 Vorträge und 57 Poster eingereicht, wobei Posterbeiträge teilweise "nur" zugeschickt werden konnten, ohne Präsenz der/des Vortragenden. Dem aktuellen Thema "Corona" wurde eine eigene Sitzung gewidmet und das Problem aus forensischer und pathologischer Sicht beleuchtet. Einen weiteren Schwerpunkt stellte die Thematik „Assistierter Suizid“ dar. Ein Novum stellten DGRM-Tagungsstipendien dar, die aufgrund der durch die Coronapandemie erschwerten Reisebedingungen an junge Wissenschaftler/-innen vergeben wurden. Der Vorstand und die Teilnehmer/-innen bedankten sich bei Michael Thali und seinem Team, trotz der neuen, sehr einschränkenden Bedingungen dafür, einen Präsenzkongress unter Pandemiebedingungen ausgerichtet zu haben - sicher und organisiert $[60,80]$.

\section{1 - Die globale Coronapandemie} dauert noch immer an. Die DGRM plante, unter Vorsitz des Tagungspräsidenten Matthias Graw (Jg. 1960) die 100. Internationale Jubiläumstagung in München, mit einer gekoppelten Festveranstaltung 
am Ort der Gründungstagung in Meran auszurichten. Zum Zeitpunkt des Redaktionsschlusses dieser Ausgabe wurde die Festveranstaltung in Meran aus Gründen der planerischen Unsicherheit abgesagt (Coronapandemie). Die 100. Tagung der DGRM soll jedoch unter eingeschränkten Bedingungen (z. B. begrenzte Teilnehmerzahl in Präsenz, virtuelle Tagungsteilnahme) im September 2021 in München ausgerichtet werden.

\section{Schlussfolgerung}

Planung, Gestaltung und Durchführung von Tagungen haben in der DGRM eine über 100 Jahre währende gute Tradition. Hierbei ging es in der Vergangenheit nicht nur um die Vermittlung wissenschaftlicher Resultate, sondern es wurden auch institutsübergreifende Probleme, insbesondere in den Eröffnungsreden der Vorsitzenden, zum Ausdruck gebracht, die im Kontext der jeweiligen Zeit standen. Die vorliegende Publikation hat nicht den Anspruch, eine eingehende Analyse der vorgestellten Tagungen vorzunehmen, sondern einen generellen Überblick über verschiedene Facetten des rechtsmedizinischen Kongresslebens zu geben. In der Betrachtung der einzelnen Kongressschriften spiegeln sich die Entwicklung des Faches, insbesondere der initiale Kampf zur Eigenständigkeit, die jeweils zeitlich bedingten wissenschaftlichen Schwerpunkte, die politischen Herausforderungen und die Möglichkeiten zukünftiger Entwicklungen wider. Bei Betrachtung der Jahreszahlen 1905 (Gründungstagung) bis 2021 (100. Jubiläumstagung) fällt eine Inkongruenz auf. Der Ausfall von Tagungen war Folge der jeweiligen allgemeinen und politischen Lage, insbesondere in Kriegs- und Nachkriegszeiten. Aber auch in heutiger Zeit können globale Probleme, wie beispielsweise die gegenwärtig noch anhaltende Coronapandemie, Einfluss auf die gewohnten Abläufe des Faches haben. Eine solche Problemlage darf jedoch nicht dazu führen, den Sinn und Zweck unseres Faches „Rechtsmedizin" aus Sicht der Versorgung und Wissenschaft zu verlieren - vielmehr sollte es immer unser Anspruch sein, Probleme, wie sie auch unsere Vorgänger/-innen im Fach zielführend angegangen sind, prak-

\section{The conferences of the German Society of Legal Medicine in the mirror of history. From Meran to Munich}

This article outlines the chronological development of the annual conferences of the German Society of Legal Medicine (DGRM) from 1905 to 2021. The medical and scientific aspects as well as the specific problems of the subject are presented in the context of the respective political and social structures and the conference culture is sketched.

\section{Keywords}

Conference · Politics - Social structures - International cooperation · Culture of remembrance

tisch, überlegt und konsequent zu lösen und über die Erfahrungen zu berichten.

Zitat Fritz Strassmann am Ende seiner Eröffnungsrede in Meran 1905 [14]:

Unsere Gesellschaft wird das sein und das bedeuten, was sie arbeitet.

\section{Korrespondenzadresse}

Prof. Dr. med. K. Albrecht

Brandenburgisches Landesinstitut für Rechtsmedizin (BLR)

Lindstedter Chaussee 6, Potsdam, Deutschland knut.albrecht@blr.brandenburg.de

Danksagung. Die Autoren bedanken sich bei Herrn Dr. med. Klaus Krocker (Berlin) für die Zurverfügungstellung von Fotografien und von Tagungsbänden (Archiv DGRM, Berlin).

\section{Einhaltung ethischer Richtlinien}

Interessenkonflikt. K. Albrecht, M. Windgassen und S. Hartwig geben an, dass kein Interessenkonflikt besteht.

Für diesen Beitrag wurden von den Autoren keine Studien an Menschen oder Tieren durchgeführt. Für die aufgeführten Studien gelten die jeweils dort angegebenen ethischen Richtlinien.

\section{Quellen}

\section{Literatur}

1. Abram R (1999) Das Kurhaus Meran. Ein Blick in die Geschichte der Kurstadt. Tappeiner, Lana

2. Herber F (2002) Gerichtsmedizin unterm Hakenkreuz. Militzke, Leipzig

3. Klimatischer Kurort Meran, Selbstverlag der Kurvorstehung. https://digital.tessmann.it. Zugegriffen:28. März 2021

4. (2019) https://de.wikipedia.org/wiki/. Zugegriffen:30.Apr. 2021

5. Krauland W (2004) Zur Geschichte der Deutschen Gesellschaft für Gerichtliche Medizin. In: Madea B
(Hrsg) 100 Deutsche Gesellschaft für Gerichtliche Medizin/Rechtsmedizin, S11-40

6. Lange-Quassowski J, Schneider V (2012) Eine bedeutende Ärztedynastie - Die Strassmanns. Hentrich und Hentrich, Berlin

7. Lignitz E (2004) Die Geschichte der gerichtlichen Medizin in der Zeit der Weimarer Republik und des Nationalsozialismus - ein Annäherungsversuch. In: Madea B (Hrsg) 100 Deutsche Gesellschaft für Gerichtliche Medizin/Rechtsmedizin, S48-72

8. Mallach HJ (1996) Geschichte der Gerichtlichen Medizin im deutschsprachigen Raum. SchmidtRömhild, Lübeck

9. Mitteilungen in: Z f Med.beamte und Krankenh.ärzte Berlin (1933), 46:363

10. Mommsen WJ (2004)DerErsteWeltkrieg.S. Fischer, Frankfurt am Main

11. Schmidt G (1986) Institut für Rechtsmedizin. In: Schettler G (Hrsg) Das Klinikum der Universität Heidelberg und seine Institute. Springer, Berlin, Heidelberg, S37-40

12. Schneider S (1983) Tagungsberichte 1951-1980 Informationen. Deutsche Gesellschaft für Rechtsmedizin Heft 23

13. Schneider V, Strassmann F (2012) Fritz Strassmann -Lebenserinnerungen

\section{Tagungsberichte (chronologisch)}

14. Vjschr. gerichtl. Med. (1906) 31:205-343

15. Vjschr. Gerichtl. Med. (1909) 37:1-180 (Suppl.)

16. Vjschr. gerichtl. Med. (1911) 41:1-272 (II. Suppl.)

17. Vjschr. gerichtl. Med. (1912) 43:1-387 (II. Suppl.)

18. Vjschr. gerichtl. Med. (1913) 43:1-361 (I.Suppl.)

19. Vjschr. gerichtl. Med. (1914) 47:1-386(Suppl.)

20. Vjschr. gerichtl. Med. (1921) 133-149

21. Dtsch.Z. ges. gerichtl. Med. (1922), 1:1-562

22. Z f Medizinalbeamte und Krankenhausärzte (1925): 5513

23. Dtsch. Z. ges. gerichtl. Med. (1927), 9: 60-67, $430,448,457$

24. Dtsch.Z.ges. gerichtl. Med. (1928), 11:408-422

25. Dtsch.Z.ges. gerichtl. Med. (1929), 13:104-391

26. Dtsch.Z. ges. gerichtl. Med.(1931), 17:131-152

27. Dtsch.Z.ges. gerichtl. Med. (1938), 29:133-332

28. Dtsch.Z.ges. gerichtl. Med. (1939), 30:258-350

29. Dtsch.Z. ges. gerichtl. Med. (1940), 32: 191-500

30. Dtsch. Z. ges. gerichtl. Med. (1952), 41

31. Dtsch.Z. ges. gerichtl. Med. (1953/54), 42:301-605

32. Dtsch.Z.ges. gerichtl. Med. (1958), 47:69-79

33. Dtsch.Z. ges. gerichtl. Med. (1959/60), 49:175-186

34. Dtsch.Z. ges. gerichtl. Med. (1959/60), 49:579-725

35. Dtsch.Z. ges. gerichtl. Med. (1961), 51:329-562

36. Acte Medicinae legalis et socialis (1961), Vol. XIV: 3-4 
37. Dtsch.Z.ges. gerichtl. Med. (1963/64), 54:1-112 38. Dtsch.Z. ges. gerichtl. Med. (1964), 55:127-264

39. Dtsch.Z. ges. gerichtl. Med. (1966), 57:1-392

40. Dtsch.Z.ges. gerichtl. Med. (1967), 59:48-344

41. Dtsch.Z. ges. gerichtl. Med. (1968), 62:52-186

42. Dtsch.Z. ges. gerichtl. Med. (1968), 64:170-172

43. Dtsch.Z. ges. gerichtl. Med. (1968), 64:165-169

44. Beitr. Ger.Med. (1974), Bd.XXXII: 1-304

45. Beitr.Ger.Med. (1975), Bd.XXXIII: 1-329

\section{Mitteilungen der Deutschen Gesellschaft für Rechtsmedizin}

46. Händel K (1993) Rechtsmedizin 3(2):R7-8

47. Weiler G (1993) Rechtsmedizin 4(1):R2-3

48. Scheithauer R (1994) Rechtsmedizin 4(3):R9

49. Saternus KS (1999) Rechtsmedizin 9(3):R10-11

50. Pollak S (2002) Rechtsmedizin 12(6):398-399

51. Pollak S (2003) Rechtsmedizin 13(6):392-393

52. Pollak S (2004) Rechtsmedizin 14(5):440-441

53. Ritz-Timme S, Bajanowski T (2005) Rechtsmedizin 15(6):499-501

54. Ritz-Timme S, Bajanowski T (2008) Rechtsmedizin 18(6):468-469

55. Ritz-Timme S, Bajanowski T (2009) Rechtsmedizin 19(6):455-456

56. Ritz-Timme S, Bajanowski T (2010) Rechtsmedizin 20(6):529-530

57. Graw M, Pfeiffer H, Ritz-Timme S (2011) Rechtsmedizin 21(6):574-575

58. Bajanowski (2015) Rechtsmedizin 25(6):591-593

59. Bajanowski (2016) Rechtsmedizin 26(6):553-554

60. Klintschar M (2020) Rechtsmedizin 30(6):480-482

\section{Tagungsprogrammhefte (chronologisch)}

61. 31.Tagung 1952, München

62. 49.Tagung 1969 , Berlin

63. 71.Tagung 1992, Berlin

64. 73. Tagung 1994, München

65. 74.Tagung 1995, Aachen

66. 75.Tagung 1996,Zürich

67. 76.Tagung 1997, Jena

68. 77.Tagung 1998, Hannover

69. 78.Tagung 1999, Frankfurt/Main

70. 79.Tagung 2000, Essen

71. 81.Tagung 2002, Rostock-Warnemünde

72. 89. Tagung 2010, Berlin

73. 91. Tagung 2012, Freiburg/Brsg.

74. 92. Tagung 2013, Saarbrücken

75. 93. Tagung 2014, Greifswald, Heringsdorf

76. 94. Tagung 2015, Leipzig

77. 96. Tagung 2017, Düsseldorf/Köln

78. 97. Tagung 2018, Halle/Saale

79. 98. Tagung 2019, Hamburg

80. 99. Tagung 2020, Zürich/ Luzern (https://www. bitzel.ch/dgrm2020/)

\section{Andreas Büttner \\ Neuropathology of Drug \\ Abuse}

Cham: Springer Nature Switzerland

2021, 3. Aufl., 120 S., (ISBN: 978-3-

030-60530-8), Softcover 85,59 EUR

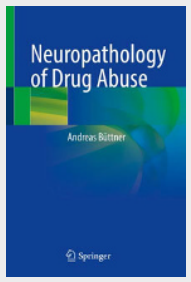

"Neuropathology of Drug Abuse" bietet auf 113 Seiten einen umfassenden Überblick über die aktuellen Erkenntnisse zu neuropathologischen Veränderungen im zentralen Nervensystem (ZNS) bei Drogenabusus. Die ersten drei Kapitel führen in die Problematik des Substanzmissbrauchs ein und geben neben neurobiologischen Erkenntnissen zur Suchtentstehung auch einen Einblick über pathologische Veränderungen des Gehirns mittels neuroradiologischer Verfahren. Der Schwerpunkt liegt auf der Charakterisierung der einzelnen Substanzen (Cannabis, Opioiden, Cocain, Amphetamin und Methamphetamin, Designerdrogen sowie neue psychoaktive Substanzen) und deren Auswirkungen auf das ZNS. Die neuropathologischen Veränderungen werden in kurzen, prägnanten Sätzen beschrieben, durch zahlreiche histologische Bilder veranschaulicht und durch eine umfangreiche Literaturangabe neuester wissenschaftlicher Publikationen ergänzt. Mit einem Ausblick über den Zusammenhang von Neurotoxizität und Neurodegeneration nach langjährigem Drogenkonsum endet das Buch mit einer kurzen Zusammenfassung und dem Ausblick auf weitere Forschungsansätze.

Simone Bohnert, Würzburg 